\title{
Consistency in solving the inverse problem of the Voce-Chaboche constitutive model for plastic straining
}

\author{
Albano de Castro e Sousa ${ }^{1}$, Ph.D. \\ Yusuke Suzuki ${ }^{2}$, Ph.D. \\ Dimitrios Lignos ${ }^{3}$, Ph.D
}

\begin{abstract}
This paper focuses on an inverse problem involving a commonly used material model in structural steel applications - the Voce-Chaboche model. In particular, this paper proposes an approach focused on obtaining a consistent set of material parameters that accurately represents a wide range of mechanical uniaxial cyclic load histories. The main focus of the methodology presented in this manuscript is its application to structural steels in the field of earthquake engineering. A set of load protocols representative of seismic loading are proposed for this purpose. It is shown that the calibration procedure optimally leverages Voce-Chaboche's ability to describe the material response to an arbitrary load history. Parameters for prevailing American, European and Japanese structural steels are also proposed. Keywords: Inverse problem, Structural steel, Chaboche, Material modeling, Earthquake loading
\end{abstract}

\section{INTRODUCTION}

Representative modeling of the behavior of structural systems at large deformations associated with collapse depends crucially on two features: material and geometric nonlinearities. Behavior is often governed by a composition of these two phenomena and, as such, greater

\footnotetext{
${ }^{1}$ Post-Doctoral Researcher, École Polytechnique Fédérale de Lausanne. EPLF ENAC IIC RESSLab, GC B3 465, Station 18, 1015 Lausanne, Switzerland. E-mail: albano.sousa@epfl.ch

${ }^{2}$ Senior Manager, Nippon Steel Corporation, Japan. E-mail: suzuki.s2k.yusuke@jp.nipponsteel.com

${ }^{3}$ Associate Professor, École Polytechnique Fédérale de Lausanne. EPLF ENAC IIC RESSLab, GC B3 485, Station 18, 1015 Lausanne, Switzerland.(corresponding author). E-mail: dimitrios.lignos@epfl.ch
} 
accuracy in modeling each of these traits will improve simulating the dynamics of their interaction. In structural steel applications this is of particular consequence in estimating member and local buckling and their performance in the post-buckling regime, which are essential in assessing behavior to collapse. This paper focuses on the material nonlinearity side of the issue.

Experimental observations of cyclically loaded structural steels show a combination of distinctive features. Chiefly among them are strain hardening, the Bauschinger effect (Bauschinger 1874), and ratcheting (Hassan and Kyriakides 1992). Strain hardening encapsulates the finding that the stress carried after some amount of irreversible deformation has a tendency to increase due to the built up resistance to the movement of dislocations caused by phenomena such as interactions with dislocations forests and dislocation pile-ups - c.f. Nabarro et al. (1964) for a classic review of the fundamental mechanisms. The Bauschinger effect captures the observation that an increase in the flow stress in a particular direction lowers the yield stress when loading is exacted in the opposite direction. This is mainly attributed to dislocation pile-ups and stress accumulation in the original load direction that when it's reversed assist the movement of the dislocations in the inverse path. Lastly, under constant stress amplitude and nonzero mean stress cyclic tests an accumulation of plastic strain with each cycle in the direction of the average stress is typically observed. This phenomenon is termed ratcheting or cyclic creep - c.f. Hassan and Kyriakides (1992) for an overview of relevant experimental results.

Modeling the aforementioned material behaviors is typically done within the $J_{2}$ plasticity framework (von Mises 1928) and literature on the subject is vast. Some focus is demanded and therefore this paper concerns itself solely with time-independent uniaxial material responses - c.f. Chaboche (2008) for a thorougher review. Time-independent material models are usually employed unless the application at hand strictly mandates a material-transient analysis. To date, structural analyses of steel buildings specific to seismic seldom consider material transient effects. Simulation strategies frequently address the aforementioned issues 
by assigning evolution laws to the yield function and the plastic potential (associative plasticity). The surfaces either change in size (isotropic hardening - Hill 1950) and/or translate in stress space (kinematic hardening - Prager 1956) in order to capture work hardening. The kinematic approach has the added feature of capturing the Bauschinger effect and as a consequence is used ubiquitously in cyclic loading. The introduction of material nonlinearity in the models can be achieved in a variety of ways: analytically, by specifying the evolution of a surface as power laws or saturating exponentials (e.g. the Ludwik 1909 or Voce (1948) expressions for isotropic hardening, respectively); a combination of linear-hardening surfaces (Hodge 1956 for isotropic and Mróz 1967 for kinematic hardening); or by stipulating growth rates as a function of the distance of the current surface to a limit surface (Dafalias and Popov 1975 and Dafalias 1986 for kinematic hardening). A significant contribution in modeling nonlinear kinematic hardening was made by Armstrong and Fredrick (1966). The simplicity in their approach in combination with its ability to simulate ratcheting has made its use widespread. Later, Chaboche et al. (1979) suggested using an additive decomposition of that rule to improve its performance. After, Dafalias et al. (2008) introduced the concept of multiplicative Armstrong-Fredrick (AF) hardening to further enhance Chaboche's model. Although AF-type laws perform well in uniaxial loading one of its main criticisms is the overestimation of ratcheting strains in multiaxial settings as shown, e.g., in Ohno and Wang (1993).

Caution should, nonetheless, be exercised when considering those criticisms as analyses are arguably only as good as the input model parameters used in performing them. As pointed out by Rezaiee-Pajand and Sinaie (2009), too often are trial-and-error approaches used to approximate a response to subjective levels of accuracy, whereas using a systematic methodology to calibrate the Chaboche model parameters can be shown to improve their behavior when compared to values previously reported in literature. Nonunique solutions is the issue at hand. Distinct parameter sets can have very similar error levels in one situation, but very disparate ones in other circumstances - c.f., e.g., Cooke and Kanvinde (2015). An 
essential part in facing this problem is, therefore, finding sufficiently differentiating conditions and minimizing the lack-of-fit of the models to the experiments. Using a diverse collection of load protocols is a valuable tool in this regard.

Past experimental studies (e.g., Krawinkler et al. 1983; Lignos et al. 2011; Suzuki and Lignos 2015) have underscored the sensitivity of structural component deterioration to the imposed loading history. Systematic load protocols have been developed for structural testing to depict the strength and stiffness deterioration of a component under seismic loading depending on the performance level of interest (e.g., Krawinkler 1996; Suzuki and Lignos 2019 ). However, there seems to be a lack of systematic protocols at a macroscopic material scale to manifest reliable material parameters (e.g., cyclic hardening) that could be used for constitutive material model input parameter calibrations to reliably prognosticate the behavior of steel structural components under seismic loading. Past experimental studies and standards (ASTM 2012; ASTM 2013; ISO 2016) have mainly focused on the identification of typical mechanical properties of structural steels under tensile loading as well as various types of fatigue applications involving constant, step-wise increasing or variable amplitude material testing (Miller and Amin 1975; Krawinkler et al. 1983; Kaufmann et al. 2001; Braconi et al. 2013; Dehghani et al. 2017) .

Methods to solve inverse problems are not straightforward and a number of suggestions to address them can be found in literature. There exist analytical procedures that are modelspecific, like the ones suggested by Rezaiee-Pajand and Sinaie (2009) or, more recently, Koo et al. (2019) which are relevant for the material model in question. Their application is however focused more on ratcheting behavior and less so on more comprehensive hysteretic responses under different load conditions. More general methodologies can be found in the context of mathematical optimization. There, one tries to minimize a well-defined objective function that describes how a material model approximates the experimental data. There are established and powerful tools under this framework for least square fitting of nonlinear functions - see e.g. Bierlaire (2015). Unconstrained gradient-based minimization methods 
like the Gauss-Newton method and its damped version, the Levenberg-Marquardt algorithm (Levenberg 1944, Marquadt 1963), are among the state of the art in inverse problems with applications in hyperelastic (Gendy and Saleeb 2000,Holzapfel et al. 2000), nonlinear heterogeneous with a prescribed stress-strain curve (Bickel et al. 2009), plastic, viscoplastic and damaged materials (Bruhns and Anding 1999, Andrade-Campos et al. 2007, Lemaitre and Desmorat 2005). A different class of methods known as randomized search algorithms can also be found in literature for the calibration of material properties, such as simulated annealing (Kirkpatrick et al. 1983), genetic and evolutionary algorithms (Pal et al. 1996, Furukawa and Yagawa 1997), and swarm algorithms (Smith et al. 2017). Although an analysis between the two approaches is beyond the scope of this paper, the reader is nonetheless referred to Andrade-Campos et al. (2007) for an interesting discussion of the topic. The scheme used in this paper is gradient-based, comparable to the Levenberg-Marquadt algorithm but formulated within a Newton Trust-Region (NTR) framework (Conn et al. 2000). Essential details on its implementation are given in the methodology section.

This paper proposes a methodology comprising (a) experimental and (b) optimization procedures in order to solve, implement, and obtain calibrated input model parameters. The material model herein consists of a nonlinear isotropic and kinematic hardening model, following the Voce and Chaboche rules, respectively. Subsequently, the results of the application of these techniques to a set European, American and Japanese structural steels will be presented, followed by a section discussing those results. The paper will finish with a brief conclusion section summarizing the main observations of this research study.

\section{METHODOLOGY}

This section presents the proposed methodology used in the inverse problem approach. It is organized into five subsections, starting with the definition materials and testing procedures, followed by selection of load protocols, a presentation on the material model, the inverse problem definition, and, finally, the recommended optimization scheme. The methodology focuses on model behavior under uniaxial load and uses this fact to simplify the evalu- 
ation procedure. The approach is based on the integration of the analytic uniaxial version of the Voce-Chaboche (VC) hardening model. The integration of the response along the strain path is conducted numerically by using discrete true-strain steps following the load protocols. It is recommended that responses be obtained from engineering metrics of uniaxial coupon tests. This approach is in contrast to procedures that explicitly model coupons in Finite Element analyses and obtain their responses by imposing displacement boundary conditions at their ends. Furthermore, this approach distinguishes itself from calibration methods that are based in far-field measurements, such as member moment-rotation responses (c.f., e.g. Araújo et al. 2017). It is believed that characterizing the response of coupon-scale specimens is a more apt descriptor of material behavior since the problem is more bounded. Member responses often carry confounding factors that are geometry-specific, such as the assumed imperfections for the member, or plastic hinge length effects that are influenced by both the cross-section geometry and material hardening (Elkady and Lignos 2018). On the contrary, tests conducted on round coupons with local strain measurements allow these problems to be significantly mitigated.

\section{Materials and testing procedures}

A number of structural steels of different grades and producers are studied in this paper - c.f. Table 1 for a summary of their tensile properties. The data analyzed herein were sourced from Grigoriou and Lignos (2017) and in-house experiments, for European steels (S355J2+N, S355J2, S460NL and S690QL) and Suzuki (2018) for typical American (A992 Gr. 50 and A500 Gr. B) and Japanese steels (BCP325, BCR295 and HYP400). Specimens were obtained from different structural elements such as plates, web and flange of I-shape sections, and the walls of square hollow sections. All specimens are uniaxially loaded. Alas, not all load protocols are available for each material type. A summary table with the material parameters is provided in the results section ( $c f$. Table 5) and the load protocols to which they were calibrated are noted explicitly. For the sake of simplicity, the analysis presented in the result section on the optimization algorithm is conducted with a standard material, 
an S355J2+N steel (nominal yield stress of 355MPa, minimum Charpy V-notch test of 27 Joules at $-20^{\circ} \mathrm{C}$ and with a normalized heat treatment) whose round coupons are extracted from $50 \mathrm{~mm}$ thick plates. The main observations, however, can be taken to hold for the whole dataset.

Testing procedures for metallic materials subject to uniaxial tensile loading at ambient temperatures have long been the subject of standardization.- c.f. e.g. ASTM (2013) and ISO (2016). Useful guiding standards for cyclic strain-controlled tests can also be - c.f., e.g., ASTM (2012). Two fundamental variables used extensively throughout this paper have to be defined: uniaxial true stress $(\sigma)$ and uniaxial true strain $(\varepsilon)$. Starting with the measured variables force $(F)$ and displacement in extensometer $(\Delta L$, with $L$ being the gauge length $\Delta L$ is the change in the initial gauge length), one defines the stress in the reference configuration as the engineering stress $\left(\sigma_{e n g}=F / A_{0}\right.$, with $A_{0}$ the initial cross-sectional area of the specimen) and the strain in the reference configuration as the engineering strain $\left(\varepsilon_{\text {eng }}=\left(L-L_{0}\right) / L_{0}\right)$. True stress and true strain are stresses and strains in the deformed configuration, which in uniaxial loading can be shown to be equal to Eq. 1 and 2, respectively - c.f., e.g. (Lubliner 2008).

$$
\begin{gathered}
\sigma=\sigma_{e n g}\left(1+\varepsilon_{e n g}\right)=\frac{F}{A_{0}}\left(1+\frac{L-L_{0}}{L_{0}}\right) \\
\varepsilon=\ln \left(1+\varepsilon_{\text {eng }}\right)=\ln \left(1+\frac{L-L_{0}}{L_{0}}\right)
\end{gathered}
$$

Challenges pertinent to the uniaxial cyclic coupon testing relate to (a) the type of testsetup grip (ASTM 2012), (b) specimen buckling, and (c)the stiffness of the test apparatus not to compound on those nonlinear geometric effects - cf. e.g. Dehghani et al. (2017).

\section{Selection of load protocols}

The uniaxial strain-based protocols are developed based on nonlinear response history analyses of more than 80 capacity-designed steel moment-resisting frames (MRFs) ranging 
from two to 12 stories. The steel MRFs comprise columns with hollow square sections (HSS) and I-shape steel beams with fully restrained beam-to-column connections. The steel MRFs are modeled in 2-dimensions (2D) in the open system for earthquake engineering simulation platform (OpenSees) (McKenna 1997). Local demand parameters (e.g., stress-strain field) is of particular interest for the load protocol development. Therefore, the use of distributed plasticity frame elements is imperative. Particularly, steel beams and columns are modeled with a force-based formulation (Spacone et al. 1996) with five integration points along their length. A fiber-based approach is employed to represent the HSS cross-section, which is discretized based on recommendations by Kostic and Filippou (2012). A uniaxial stress-strain formulation is assigned to each one of the fiber elements based on the Menegotto-Pinto uniaxial formulation (Menegotto and Pinto 1973). The input model parameters are calibrated to available test data (Lignos and Krawinkler 2013). The component modeling does not consider the effects of strength and stiffness deterioration on the structural response. Arguably, component deterioration is likely to dominate the steel MRF response at large inelastic drift demands (Zareian et al. 2010). However, this is not expected to be the case at modest drift demands associated with design-basis seismic events with a probability of exceedance of $10 \%$ in 50 years or in cases that P-Delta effects dominate the structural response prior to collapse (Adam and Jäger 2012). Therefore, the modeling approach is justified. Second order effects are considered with the P-Delta transformation in OpenSees. Damping is simulated with the Rayleigh model according to the recommendations of Zareian and Medina (2010). A two percent $(2 \%)$ viscous damping ratio is considered for the fundamental period, $T_{1}$ of each frame and for that corresponding to $20 \% T_{1}$ as suggested in PEER (2010). The steel MRFs are subjected to suites of 40 ground motions. Each of which representing near-fault, long duration (from subduction interfaces) and ordinary seismic records based on the conditional mean spectrum (Baker 2011). Specifics to the ground motion selection are discussed in Suzuki and Lignos (2019). While seismic response at design-basis earthquakes is of interest, the ordinary ground motion set is scaled incrementally till collapse occurs only steel MRFs 
vulnerable to P-Delta (i.e., above 8-stories) in an attempt to get a sense of column strain demands through collapse, acknowledging the limitations of the employed numerical models.

The simulation results of interest feature uniaxial strain histories extracted from the outer extremity of the flat part of the first-story end (exterior) column of a steel MRF in the principal direction of earthquake loading. Results indicate that for ordinary and longduration records, mean effects are negligible. Therefore, representative strain-based load protocols are developed based on standard rainflow-counting with zero mean effects (ASTM 2011). In near fault records, two distinct types of strain responses are dominant. Those that a member exhibits a full-cycle of a certain strain amplitude followed by monotonic drifting in one loading direction prior to collapse due to P-Delta effects (i.e., typical in steel MRFs above 8-stories) as well as cases that while collapse does not occur mean effects are appreciable. These two types are treated separately. In the former, the absolute peak strain demand prior to collapse is identified for steel MRFs with 8 or more stories. In the latter, rainflow-counting is conducted two times. The first one is up to the absolute peak strain demand, whereas the second one is conducted in the post-peak strain response up to rest. Conservatively, the 90th percentile of the cumulative frequency distribution of the acquired strain demands is utilized in all cases to establish the strain-based load protocols. This is consistent with the methodology presented in Krawinkler et al. (1983).

\section{Material model}

This subsection presents a nonlinear isotropic and kinematic hardening model within the framework of $J_{2}$ plasticity. The essential features of its integration procedure can be readily consulted in its open-access implementation RESSPyLab (de Castro e Sousa et al. 2019). For a thorougher discussion on the implementation of inelastic materials the reader is referred to Simo and Hughes (1998).

From the $\pi$-plane perspective the threshold for plasticity, as defined by the Von Mises yield criterion, can be seen as a circle and isotropic hardening can be interpreted as an increase of the radius of the circle, and kinematic hardening a translation movement of the 
center of that circle.

Dictating how this circle enlarges and moves as function of the loading is commensurate to defining material hardening laws. Finding appropriate parameters for those rules is the subject of this paper. Henceforth the uniaxial version of the problem will be presented since the analysis of test-data will be solely unidirectional. Consider the isotropic and kinematic hardening laws in Eq. 3, 4 and 5.

Isotropic hardening (Voce 1948)

$$
\sigma_{y}=\sigma_{y, 0}+Q_{\infty}\left(1-e^{-b \varepsilon_{e q}^{p}}\right)
$$

Kinematic hardening (Chaboche et al. 1979)

$$
\begin{aligned}
\dot{\alpha}_{k} & =\operatorname{sign}(\sigma-\alpha) C_{k} \dot{\varepsilon}_{e q}^{p}-\gamma_{k} \alpha_{k} \dot{\varepsilon}_{e q}^{p} \\
\alpha & =\sum_{k=1}^{n B a c k} \alpha_{k}
\end{aligned}
$$

where $\varepsilon_{e q}^{p}$ is the equivalent plastic strain whose definition for uniaxial loading can be expressed as in Eq. 6.

$$
\dot{\varepsilon}_{e q}^{p}=\left|\dot{\varepsilon}^{p}\right|
$$

Equation 3 is an exponentially saturating isotropic hardening law, i.e. as plastic strain is accumulated the increase in stress will tend to the maximum saturated value $Q_{\infty}$ above yield. It will do so governed by the exponential term at a rate defined by the term $b$.

Equation 4 is a kinematic hardening rule defined in rate form. There are two notable terms: (1) the term with the parameter $C_{k}$ if it existed by itself would lead to a simple linear kinematic hardening model and (2) the term with $\gamma_{k}$ is negatively proportional to the backstress itself and, thus, leads to a nonlinear exponentially saturating behavior. The 
introduction of the $\gamma_{k}$ term was suggested by Armstrong and Fredrick (1966) and so this rule bears their name. For monotonic loading Eq. 4 has essentially the same form of Eq. 3 where the saturation term is $C_{k} / \gamma_{k}$ and the rate term is $\gamma_{k}$. After Armstrong and Fredrick (1966), Chaboche et al. (1979) suggested the use of multiple backstresses (c.f. Eq. 5) to the define a more robust rule.

With the material parameters discussed above, one can give the formal definition of the uniaxial Von Mises yield criteria used to solve the inverse problem in Eq. 7, in which $\boldsymbol{\theta}$ is a vector containing all the model parameters.

$$
\phi^{V M}\left(\sigma, \alpha, \sigma_{y} ; \boldsymbol{\theta}\right)=\phi^{V M}\left(\sigma, \alpha, \sigma_{y} ; E, \sigma_{y, 0}, Q_{\infty}, b, C_{1}, \gamma_{1}, \ldots, C_{k}, \gamma_{k}\right)=(\sigma-\alpha)^{2}-\sigma_{y}^{2} \leq 0
$$

\section{Inverse problem definition}

All optimization procedures operate on an objective function to be minimized. In the particular case of inverse problems, a measure of fitness between a model and an experimental result is sought after. For classic plasticity problems a number of different objective functions have been used for this purpose - c.f. Pal et al. (1996) and Smith et al. (2017). What follows presents and discusses what is believed to be a robust alternative to those sources.

Consider the definition of the accumulated true strain in Eq. 8, which expresses the sum of the absolute values of the strain increments for a given load protocol. The purpose of this variable is to be able, for each protocol, to define a loading function in a strict sense where one input $\left(\varepsilon^{*}\right)$ has a unique corresponding output $(\sigma)$. Referring to Fig. 1, or cyclic loading, this is equivalent to "unpacking" the hysteretic true-stress-true-strain curve.

$$
\varepsilon^{*}=\int_{0}^{t}|\dot{\varepsilon}| d \tau
$$

Equation 9 presents the error function to be minimized in the inverse problems. The $i$-th load protocol is expressed by the variable $\varepsilon_{i}^{*}$ of a load protocol set of $n_{\text {Tests }}$ number of 
tests. The numerator consists of the sum over all experimental results (e.g. whose main variable is ) of the integral of the square difference between the model and experimental true stress over the accumulated true strain. This is tantamount to the square of the grey shaded area in Fig. 1b. The denominator is an averaging term to provide an error per unit strain increment. Nonlinear least squares is akin to this approach in that it is a discrete sum of square differences. Here, however, a continuous representation is used with an integral along the loading path, albeit in practice it is numerically integrated with the trapezoidal rule. Another possibility would be to use the Least Absolute Deviation (LAD). This approach is sometimes used because it is both computationally efficient and is known to be less sensitive to outliers (Wilson 1978). However, since differentiability near the solution is a desirable feature in methodologies that rely on gradient computations and since a procedure of this class is used herein, the squared difference is used.

$$
\varphi\left(\varepsilon_{i}^{*} ; \boldsymbol{\theta}\right)=\sum_{i} \frac{\int_{0}^{\varepsilon_{i}^{*}}\left(\sigma_{\text {model }}\left(\varepsilon_{i}^{*} ; \boldsymbol{\theta}\right)-\sigma_{\text {Test }}\right)^{2} d \epsilon^{*}}{\int_{0}^{\varepsilon_{i}^{*}} d \epsilon^{*}} \quad, \quad i \epsilon\left\{1, \ldots, n_{\text {Test }}\right\}
$$

To avoid negative solutions an additional reciprocal barrier equal to $1 / \theta_{i}^{2}$ is added to $\varphi$, with $\boldsymbol{\theta}$ the model parameters and steps are restricted to within $\forall i, \theta_{i} \geq 1 e-4$.

The error function in Eq. 9 can be challenging to interpret (units of $M P a^{2}$ ) and so a normalized error function in Eq. 10 will be used to facilitate the discussion of the results. It represents the square root of the ratio between the integral of squared difference and just the squared area underneath the test.

$$
\bar{\varphi}=\sqrt{\varphi /\left\{\sum_{i} \frac{\int_{0}^{\varepsilon_{i}^{*}}\left(\sigma_{T e s t}\right)^{2} d \epsilon^{*}}{\int_{0}^{\varepsilon_{i}^{*}} d \epsilon^{*}}\right\}}
$$

\section{Optimization procedure}

The algorithm is implemented in the Python package RESSPyLab (de Castro e Sousa et al. 2019) for solving the inverse problem in present paper.

To illustrate the methodology, consider the second-order Taylor approximation $m$ of some 
function $f\left(f: \Re^{2} \rightarrow \Re\right.$, whose level curves are represented in Fig. 2a) about a point at the $k$-th iteration $\left(\mathbf{x}_{k}\right)$ in Eq. 11, with $\mathbf{d}$ the difference between the point at the next step and the current one $\left(\mathbf{d}=\mathbf{x}_{k+1}-\mathbf{x}_{k}\right), \nabla_{\mathbf{x}} f$ the gradient of $f$, and $\nabla_{\mathbf{x} \mathbf{x}} f$ the Hessian of $f$.

$$
f\left(\mathbf{x}_{k+1}\right) \approx m\left(\mathbf{x}_{k+1}\right)=m\left(\mathbf{x}_{k}+\mathbf{d}\right)=f\left(\mathbf{x}_{k}\right)+\nabla_{\mathbf{x}} f\left(\mathbf{x}_{k}\right)^{T} \mathbf{d}+\frac{1}{2} \mathbf{d}^{T} \nabla_{\mathbf{x x}} f\left(\mathbf{x}_{k}\right) \mathbf{d}
$$

If one were to simply apply Newton's method into finding the minimum of $m$, one would look for the step $\mathbf{d}$ that would yield $\nabla_{\mathbf{d}} m=0$. From Eq. 11, this results in Eq. 12, which finds the minimum of $m$ for positive-definite Hessians in a single step.

$$
\nabla_{\mathbf{d}} m=\nabla_{\mathbf{x}} f\left(\mathbf{x}_{k}\right)+\nabla_{\mathbf{x} \mathbf{x}} f\left(\mathbf{x}_{k}\right) \mathbf{d}=0 \Rightarrow \mathbf{d}=-\nabla_{\mathbf{x x}} f\left(\mathbf{x}_{k}\right)^{-1} \nabla_{\mathbf{x}} f\left(\mathbf{x}_{k}\right)
$$

The problem with taking this step is that far from $\mathbf{x}_{i}$ the quadratic approximation might not at all be representative of $f$. To illustrate this point consider the search direction delineated in dashed stroke in Fig. 2a and 2b, which is depicted in Fig. 3a. To prevent this, the size of step $\mathbf{d}$ is restricted to a certain magnitude $(\Delta)$ of a specified $l$-norm $\left(\|\mathbf{x}\|_{l}=\right.$ $\sqrt[l]{\sum_{j}\left|x_{j}\right|^{l}}$; cf. Fig. 3b ) where one is confident that $f \approx m$ - the Trust-Region.

The next step in the methodology is how to find the step $\mathbf{d}$ that minimizes $m$ such that $\|\mathbf{d}\|_{l} \leq \Delta$ - the trust-region sub-problem. This is formalized by the optimization problem 13 with constraints 14 .

$$
\begin{array}{ll}
\text { minimize } & m\left(\mathbf{x}_{k}+\mathbf{d}\right) \\
\text { subject to } & \|\mathbf{d}\|_{l} \leq \Delta
\end{array}
$$

The analyses conducted in this article will use the 2-norm with the Steihaug-Toint truncated conjugate gradient method (Bierlaire 2015). 
To assess how much the path should be restricted, a metric on the quality of the secondorder approximation is usually employed. The ratio between the change in the value of the function and the approximation due to the step $\mathbf{d}$ is often used for this purpose - c.f. Eq. 15.

$$
\rho=\frac{f\left(\mathbf{x}_{k}\right)-f\left(\mathbf{x}_{k}+\mathbf{d}\right)}{m\left(\mathbf{x}_{\mathbf{k}}\right)-m\left(\mathbf{x}_{k}+\mathbf{d}\right)}
$$

Ideally this assessment should be revised as the search space is traversed. A two parameter criterion $\left(\eta_{1}\right.$ and $\left.\eta_{2}\right)$ is usually employed to judge the update of the step size $\Delta$. If $\rho \geq \eta_{1}$ then $\Delta$ is increased, whereas if $\rho \leq \eta_{2}$ then $\Delta$ is decreased. Between these two values $\Delta$ remains unchanged. Take, for instance, $\eta_{1}=0.9$ and $\eta_{2}=0.01$, this means that if the approximation fits upwards of $90 \%$ the function value, then it is deemed to be sufficiently good so that the restriction on the step size is relaxed. The converse happens when it fits to less than $1 \%$ of the function value (Conn et al. 2000, Bierlaire 2015).

One important detail about the Steihaug-Toint truncated conjugate gradient procedure is that in the event of an indefinite Hessian, it leverages valuable properties of the method by conducting Hessian-orthogonal steps in descent directions. Since they are bounded by the trust region one does not risk an unbounded search and, by always checking the quality of solution with Eq. 15, it provides a reliable criterion for effectively canvasing the objective function's landscape. Additionally, it is worth noting that the presence of indefinite Hessians usually points to the existence of multiple local minima. One possible strategy to further explore the variable space in the search for a global minimum is to keep track of the points where negative eigenvalues occur and generate search paths on opposite senses of the negative eigenvector - c.f. Wales (2003).

A potential problem in the convergence rate of the algorithm happens when the condition number of the Hessian $\nabla_{\mathbf{x} \mathbf{x}} f\left(\mathbf{x}_{k}\right)$ is large. A solution to mitigate this issue is to solve a preconditioned sub-problem. The idea is not to restrict step $\mathbf{d}$ to some magnitude, but apply that constraint on a distorted version of $\mathbf{d}$ (defined as $\mathbf{w}$ ), which is carefully chosen 
to follow the local curvature of the quadratic model. This is more formally expressed in Eq. 16 , where $\mathbf{S}$, to follow the curvature, should precondition as much as possible the Hessian of the model. An illuminating discussion of this topic can be found in Conn et al. (2000) under the rubric of Trust-Region Scaling.

$$
\mathbf{S}_{k} \mathbf{w}=\mathbf{d}
$$

From Eq. 16, one can thus define a preconditioned model of $f$ around $\mathbf{x}_{k}$ as $m^{P}$ as shown in Eq. 17

$$
f\left(\mathbf{x}_{k}+\mathbf{d}\right)=f\left(\mathbf{x}_{k}+\mathbf{S w}\right) \approx m\left(\mathbf{x}_{k}+\mathbf{d}\right)=m^{P}\left(\mathbf{x}_{k}+\mathbf{w}\right)
$$

Which from Eq. 11 and 16 yields Eq. 18.

$$
m^{P}\left(\mathbf{x}_{k}+\mathbf{w}\right)=f\left(\mathbf{x}_{k}\right)+\left(g_{k}^{P}\right)^{T} \mathbf{w}+\frac{1}{2} \mathbf{w}^{T} \mathbf{H}_{k}^{P} \mathbf{w}
$$

with,

$$
\begin{array}{r}
g_{k}^{P}=\mathbf{S}_{k}^{T} \nabla_{\mathbf{x}} f\left(\mathbf{x}_{k}\right) \\
\mathbf{H}_{k}^{P}=\mathbf{S}_{k}^{T} \nabla_{\mathbf{x x}} f\left(\mathbf{x}_{k}\right) \mathbf{S}_{k}
\end{array}
$$

Thus, given a certain preconditioner $\mathbf{S}_{k}$, one can now solve the equivalent sub-problem in Eq. 21 for $\mathbf{w}$.

$$
\begin{array}{ll}
\text { minimize } & m^{P}\left(\mathbf{x}_{k}+\mathbf{w}\right) \\
\text { subject to } & \|\mathbf{w}\|_{l} \leq \Delta
\end{array}
$$


To guide the choice of a proper preconditioner, it is often helpful to think of performing the change of variables geometrically, where it would be natural to state that one would wish to work on a model where its eigenvalues have equal magnitude. The clearest way to achieve this would be to impose the Hessian of the scaled model to be equal to the identity matrix - Eq. 22.

$$
\mathbf{I}=\mathbf{H}_{k}^{P}=\mathbf{S}_{k}^{T} \nabla_{\mathbf{x x}} f\left(\mathbf{x}_{k}\right) \mathbf{S}_{k} \Leftrightarrow \nabla_{\mathbf{x} \mathbf{x}} f\left(\mathbf{x}_{k}\right)=\left(\mathbf{S}_{k} \mathbf{S}_{k}^{T}\right)^{-1}
$$

If the Hessian of $f$ is locally symmetric positive-definite, a natural method for computing $\mathbf{S}_{k}$ would be to use the Cholesky decomposition as it is commonly done in the preconditioned conjugate gradient method - c.f. Bierlaire (2015). A more general approach would be to impose that $\mathbf{S}_{k}$ is itself symmetric, which would yield Eq. 23.

$$
\nabla_{\mathbf{x x}} f\left(\mathbf{x}_{k}\right)=\left(\mathbf{S}_{k} \mathbf{S}_{k}^{T}\right)^{-1}=\left(\mathbf{S}_{k}^{2}\right)^{-1} \Leftrightarrow \mathbf{S}_{k}=\left(\nabla_{\mathbf{x x}} f\left(\mathbf{x}_{k}\right)\right)^{-\frac{1}{2}}
$$

Computing $\mathbf{S}_{k}$ in Eq. 23 can then be done, similarly to the calculation of Moore-Penrose pseudoinverses, with the Singular Value Decomposition (SVD) of the Hessian of $f$ - c.f. Eq. 24.

$$
\mathbf{S}_{k}^{S V D}=\left(\nabla_{\mathbf{x x}} f\left(\mathbf{x}_{k}\right)\right)^{-\frac{1}{2}}=\left(\mathbf{U}_{k} \boldsymbol{\Sigma}_{k} \mathbf{V}_{k}^{T}\right)^{-\frac{1}{2}}=\mathbf{V}_{k} \boldsymbol{\Sigma}_{k}^{-\frac{1}{2}} \mathbf{U}_{k}^{T}
$$

where $\mathbf{U}_{k}$ and $\mathbf{V}_{k}$ are matrices that store the left and right singular vectors, respectively, and the matrix $\boldsymbol{\Sigma}_{k}$ stores the singular values along its diagonal.

The disadvantage of using this approach is the additional computational cost involved in computing the SVD of the Hessian, which may or may not be make up for itself in an increased rate of convergence of the overall algorithm.

Performing an SVD is an ideal situation, an upper bound, so to speak, for the preconditioner $\mathbf{S}_{k}$. The converse would be a transformation that leaves everything unchanged with $\mathbf{S}_{k}=\mathbf{I}$ so that $m^{p}=m$. In between there could be any number of preconditioners, which 
will approximate the Hessian to greater or lesser degrees in the sense of Eq. 22.

One commonly used, and computationally cheap, way of the approximating the Hessian is the so called Jacobi preconditioner ( $c f$. Eq. 25). This method considers that the diagonal elements of the Hessian provides a sufficiently accurate approximation of this matrix, while being easily invertable.

$$
\mathbf{S}_{k}^{J a c o b i}=\operatorname{diag}\left(\nabla_{\mathbf{x x}} f\left(\mathbf{x}_{k}\right)\right)^{-\frac{1}{2}}
$$

By taking only the diagonal elements of the Hessian it is assumed they are representative of most of the magnitude of the eigenvalues, as such this preconditioner could be more aptly described as a scaling transformation.

As can be seen in Eq. 11, the NTR method relies heavily on the ability to compute the gradient and the Hessian of the objective function. When it comes to the class of inverse problems, which is the focus of this report, derivatives have to be computed on the integration scheme over the parameters of the model. This can be quite challenging and one will perform analyses using two methodologies on the objective function: (1) numerical differentiation (ND) with central differences and (2) algorithmic (or automated) differentiation (AD). The former approach is quite standard, but is known to be handicapped by truncation and rounding errors depending on the step size. The ND analyses presented herein were made using the central difference method with a fixed step size of $1 e-6$. The AD approach is the result of the systematic application of chain rule over the graph of a user-defined computer function, which returns derivatives with a high level of accuracy - c.f. Griewank and Walther (2008). A Python package called numdifftools (Brodtkorb 2017) was used that implements both approaches. This package is based on the Matlab program written by D'Errico (2006) and also wraps around the package AlgoPy (Walter and Lehmann 2013) for algorithmic differentiation.

The results shown in the following section are a direct application of this methodology to the minimization procedure of the inverse problem expressed in Eq. 9 with respect to 
parameter vector $\boldsymbol{\theta}$. The choice of the initial starting point for the NTR algorithm can have a significant influence on the solution. In this report, the criterion for the starting point (S.P.) in most NTR optimizations was chosen so as to have an elastic-perfectly-plastic model with the yield stress equal to the nominal yield value of the steel $\hat{\sigma}_{y, 0}$ and with the nominal elastic modulus $\hat{E}$. All other parameters were set to a residual value of $1 e-1$, which has minimal impact both on the perfectly-plastic assumption and on the barrier added to $\varphi$. The results for other starting points is presented in sub-section 3. One of these solution points is the extreme case where very little except the nominal value of modulus of elasticity is known, i.e. $\sigma_{y, 0} \approx 0$. In addition, results for an intermediate starting point at $\sigma_{y, 0}=0.5 \hat{\sigma}_{y, 0}$ are also presented.

\section{Load protocol sampling}

Since the model should ideally capture material response irrespective of the imposed history, the question naturally follows of how sensitive is the calibration when using a different load protocol set. To address this question, one needs to define a metric that distinguishes two solutions with respect to each other. It is also useful to define a base case to which all comparisons are made. The base case chosen for all analyses is the maximum number of available load protocols since it contains the maximum amount of information on the problem. Other cases will be samples of that set. Due to the inordinate number of possible sample sets for only 10 total load protocols, a small number of sets (50) were selected with the guiding principle of (1) having a low number of load protocols and (2) having distinct groups of load protocols of small and large amplitude histories. The first focuses on the desire to know what is the minimum number of load protocols necessary to achieve an acceptable accuracy, and the second on the influence of the amplitude magnitude on the error function.

Equations 26 and 27 represent in a sense two different metrics to evaluate a distance between two points. $\xi_{1}$ is the most intuitive choice as it is merely the root of the sum of the relative errors squared of each of the model parameters. $\xi_{2}$ is less obvious and is best understood in conjunction with Eqs.10 and 12. From Eq. 12, the numerator within the 
root of Eq. 27 represents the increase in the quadratic approximation of the error function from the base point. The denominator in Eq. 27 is used for the same reason as in Eq. 10, i.e. to provide a normalizing term. Now since at the base point we have a positivedefinite Hessian, any deviation from $\boldsymbol{\theta}_{\text {base }}$ will increase monotonically and, therefore, the distance to any minima obtained with a different protocol set is a positive metric weighed by the objective function itself. The usefulness of $\xi_{2}$ will become apparent in the discussion section, particularly while analyzing solutions with a large number of backstresses where the positive-definite condition is technically satisfied albeit with very small eigenvalues. In such situations there are solutions which maybe far from each other in the $\xi_{1}$ sense but which do not have a significant impact in evaluating the error function with respect to the base case. When this happens the two solution points are said to be consistent with each other.

\section{RESULTS}

\section{Load protocols}

Figure 4 shows the derived strain protocols for macroscopic material evaluation of structural steels for cyclic loading representative of seismic applications. The reference protocols are complemented by monotonic one (see Figure 4a) conducted according to ASTM specifications. The test should be carried out to fracture of the specimen. Figure $4 \mathrm{~b}$ depicts representative strain protocols with monotonic tension after a full cycle of cyclic inelastic straining. Low-rise steel MRFs subjected to near-fault records are likely to experience a large pulse prior to drifting in one loading direction. The strain range in HSS columns in this case is $\Delta \varepsilon=10 \%$ whereas the equivalent one in high-rise steel HSS columns is $\Delta \varepsilon=2 \%$ prior to the monotonic tensile push to fracture. Figure 4e shows a second protocol corresponding 
to strain demands representative of near-fault records when collapse does not occur. The rupture directivity, characteristic of near-fault records, is depicted by the impulsive doublesided strain cycle with $3 \%$ amplitude. In long duration records, it is found that constant strain amplitude tests of at least 20 full cycles represent fairly well the seismic demands in HSS columns in steel MRFs. Particularly, strain amplitudes of $1 \%$ and $2 \%$ are found to represent, on average, strain demands in mid-to-high-rise and low-rise steel MRFs, respectively, subjected to subduction zone seismic events as depicted in Figure 4c. During these tests, the material is expected to reach a steady state response, thereby exhibiting stress saturation. Incremental load protocols up to a $2 \%$ uniaxial strain demand (i.e., strain range $\Delta \varepsilon=4 \%$ ) are deemed reasonable to represent, on average, seismic demands in steel HSS columns during design-basis earthquakes. To consider the influence of a steel MRF's height (i.e., predominant period) on the developed protocols, rain-flow counting is benchmarked to the results of the 2- 6- and 12-story steel MRFs. Figure 4d shows the derived protocols. Strain demands above $2 \%$ until failure (i.e., buckling or fracture) is an arbitrary decision in case the material may reach stress saturation. Referring to Fig. 4f, a protocol representative of column strain demands prior to steel MRF collapse is shown. While non-symmetric strain demands are evident, which is a characteristic of response histories prior to earthquake-induced collapse (Lignos et al. 2011), the small strain reversals are not expected to shift a material's behavior that much from a conventional monotonic tensile test.

\section{Optimization algorithm performance}

All analyses in this subsection are demonstrated using an S355J2+N steel sampled from $50 \mathrm{~mm}$ thick plates. The full collection of load protocols (LP) introduced in the previous section are used in solving the inverse problem (1 to 10).

\section{Numerical and Algorithmic Differentiation in NTR}

Figure 5 shows a comparison between ND and AD for a Jacobian-preconditioned algorithm $(\mathrm{J})$ with one backstress. Both analyses use as a starting point an elastic-perfectly plastic model, plateauing at the steel's nominal yield stress. Here, the imprecision associ- 
ated with ND in combination with scaling that happens during preconditioning, can lead the algorithm to get locked in to searching for solutions in directions where $\varphi$ decreases at a very slow rate. This underlines the importance in securing an accurate derivative estimation, for which AD is a useful tool.

Hence, all results in subsequent analyses will use algorithmic differentiation.

\section{Preconditioning in NTR}

Figure 6 shows a comparison between no preconditioning, Jacobi $(\mathrm{J})$ and singular value decomposition (SVD) preconditioning of the NTR Steihaug-Toint sub-problem for a 2 backstress model. Here, again, the analyses use as a starting point an approximately elasticperfectly plastic model, plateauing at the steel's nominal yield stress. As can be seen, the cost of not using a preconditioner can be prohibitively high. This is more noticeable the more backstresses are used in the calibration. Also, the Jacobi and SVD preconditioning yield similar convergence rates although, from experience, the SVD is more stable numerically near the solution.

\section{Different starting points and number of backstresses}

All previous analyses were conducted with the starting point of an approximately elasticperfectly plastic material with nominal yield stress and elastic modulus. In this sub-section two other starting points are investigated, for a total of three starting values:

- Starting point 1 (S.P. 1): $\sigma_{y, 0}=\hat{\sigma}_{y, 0}=355 M P a$

- Starting point 2 (S.P. 2): $\sigma_{y, 0}=0.5 \hat{\sigma}_{y, 0} \approx 178 M P a$

- Starting point 3 (S.P. 3): $\sigma_{y, 0}=1 e-1 \approx 0 M P a$

The algorithm used for the optimization procedure in all cases was the NTR with AD, Jacobi and SVD preconditioning. Results for 2 backstresses are presented terms of the solution path and final true stress-true strain curves for all load protocols in Figs. 7 and 8, respectively. Tables 2 and 3 present a summary of the algorithm's performance and its solution point at convergence. Since the results for both J and SVD preconditioning are the same, 
the table is condensed to include solely the SVD results. Furthermore, a comparison between experimental and model true-stress-true-strain responses with the parameter solution for 2 backstresses is given in Fig. 8 .

Referring to Fig. 7 and Table 3, it can be readily observed that for one, two, and three backstresses that same solution(model parameters) is reached regardless of the starting point. For four backstresses, for virtually the same accuracy in the error function, two different solution points were retrieved, confirming the nonuniqueness issue often debated in inverse problems (Cooke and Kanvinde 2015).

It was also found that the relative decrease in error using more backstresses, even for the wide variety of load protocols involved, is negligeable after a certain point. The error associated with two backstresses was found to be virtually the same as three and four backstresses.

\section{Load protocol sampling}

Table 4 shows results for the calibration S355J2+N steel Voce-Chaboche material parameters for different samples from the full set of 10 load protocols (Set 1) with two backstresses. The parameters obtained are compared to the normalized error $(\bar{\varphi}) \operatorname{computed}$ with the full set of protocols to judge their relative impact. The results are ordered in ascending order of this metric. Also shown in Table 4 are the distances $\xi_{1}$ and $\xi_{2}$ defined in Eqs. 26 and 27, respectively, with respect to the base parameters of Set 1.

\section{Summary of results for different structural steel materials}

The results presented previously were obtained for an S355J2+N steel. Notwithstanding, the same methodology was applied to a a broad range of European, American and Japanese steels with the objective of obtaining material parameters for reference and comparison purposes. For context, a summary of the tensile properties for those materials is given in Table 1, and Table 5 catalogs the acquired results for the Voce-Chaboche model with 2 backstresses. The implications of the obtained parameters are expounded in the Discussion section. 


\section{DISCUSSION}

\section{Algorithm performance}

In order to achieve acceptable convergence rates there are two important factors that should be fulfilled in order to use NTR with the Steihaug-Toint method: (1) accurate derivative estimates and (2) hessian preconditioning. The first point can be clearly inferred from Fig. 5 where algorithmic differentiation is compared with the more imprecise approach of a fixed step central difference numerical differentiation. Although the convergence rate could be improved by using an adaptive step size, the computational cost associated with this option is high and, therefore, does not justify itself over the use of algorithmic differentiation. In addition, algorithmic differentiation has the benefit of being generally applicable to different hardening laws. Consequently, instead of painstakingly calculating derivatives that result from the integration algorithm of a specific material law, algorithmic differentiation achieves high accuracy levels with minimal implementation effort at a reasonable computational cost and is, thus, a valuable tool to employ. With respect to the second point, greater rates of convergence are achieved using a preconditioned hessian (c.f. Fig. 6) in large part due to the use of the conjugate gradient method in the Steihaug-Toint procedure (Conn et al. 2000). This effect is evermore present the greater the condition number of the hessian of the problem - i.e. the ratio of its highest to lowest eigenvalues. In this particular inverse problem, this observation is more noticeable the higher the number of backstresses used. As for the preconditioner itself, the two options presented in this study (Jacobi and SVD) have similar convergence rates. Both are presented because while the SVD is more stable numerically, its computational cost can be prohibitive for models with a higher number of parameters. In these cases the Jacobi preconditioner can be a useful alternative.

\section{Nonuniqueness and consistency}

The suitability of working with the NTR method in solving the VC inverse problem should also be evaluated with respect to the possibility of retrieving multiple solutions in the parameter space, as observed in the results presented in Table 2. This possibility depends 
both on the nonlinearity of the underlying material model and on the error function 's definition. Due to the multi-dimensional nature of the problem, it is challenging to provide a geometric depiction of the error function's landscape. One can, nonetheless, have an idea of the nonlinear nature of the problem by tracking the evolution of its eigenvalues. More concretely, if one inquires into the sign of the minimum eigenvalue of the Hessian, one can immediately get the sense if locally along the path to the minimum one encounters what resembles hyperbolic paraboloids (saddle points). Figure 9 shows that evolution for one and four backstresses at two different starting points. It can be seen that hyperbolic paraboloids are indeed encountered in the NTR path. This type of nonlinearity is particularly salient in the four backstress model where the Hessian switches from positive-definite to indefinite multiple times. Naturally, all models finish with a positive-definite Hessian thus ensuring, along with a strict tolerance on the gradient (e.g. $\|\nabla \varphi\|<1 e-10$ ), a proper minimum. Although the presence of negative eigenvalues is suggestive of multiple minima, it does not necessarily imply nonunique solutions since a number of nonconvex examples can be produced that posses solely one global and local minimum. General statements regarding solution uniqueness of the $\mathrm{VC}$ inverse problem, therefore, cannot be made.

From the discussion in the previous paragraph, one hopes that the nonlinear nature of the problem is clear to the reader. In light of this fact it is quite astonishing to find that the solutions retrieved with NTR are generally close to each other, although the path they take to reach their values are significantly different. The results presented in Fig. 7 illustrates this fact, as shown in the evolution of the parameters with respect to the algorithms' iterations. Though anecdotal in context, it underscores the robustness of the proposed methodology.

The impossibility to make general statements regarding nonuniqueness notwithstanding, one can deploy other metrics to evaluate the consistency of the solutions. Specifically, one can compare the cases relative to each other using the metrics $\xi_{1}$ and $\xi_{2}$ described in the Methodology section. Take, for example, the four backstresses cases where three different starting points yielded three different solutions amid keeping the normalized error function 
values essentially the same - c.f. Table 2 . The solutions are appreciably different particularly in terms of the magnitude of their $C_{k}$ parameters. In this case one can, in fact, use the error metrics introduced in Eqs. 26 and 27 to quantify objectively the differences between the solutions. For this purpose let starting point 1 serve as the base case. $\xi_{1}$ for starting points 2 and 3 is $\approx 22.71 \%$ and $\approx 5.75 \%$ respectively, while $\xi_{2}$ is $\approx 1 e-6 \%$ for both cases. These results have important implications in interpreting different calibrations. Whereas, $\xi_{1}$ shows a noticeable difference between parameters, $\xi_{2}$ suggests that those differences are small when assessing the error function with respect to the base case. It is crucial here to recall that the $\xi_{2}$ metric increases monotonically from the minimum if the Hessian is positive-definite. Such small values as $1 e-6$ for $\xi_{2}$ essentially implies that, although numerically the Hessian might be positive-definite, the relative direction between the two solutions lies close to what can practically be described as the null-space of the Hessian. Now, choosing solutions that locally around the base case (in the $\xi_{2}$ sense) do not significantly increase the error function implies that those points predict behavior similarly and, for this reason, are said to be consistent with each other. A more debatable issue is defining an acceptable threshold value for the local increase in the error function. More on this topic is discussed in the load protocol sampling section.

\section{Load protocol sampling}

Table 4 shows results for analyses conducted with samples from a set 10 load protocols ordered by the relative error $\bar{\varphi}$ that the parameters of the subset have when considering all the load protocols. In conjunction with $\xi_{1}$ and $\xi_{2}$, one can also evaluate their level of nonuniqueness and consistency.

With respect to $\bar{\varphi}$, most of the solutions of the sampling sets have errors that are close to full set. Notable among them are sets 45 and 18, whose solutions provide essentially the same $\bar{\varphi}$ but with three load protocols as opposed to 10. Equally interesting is that the load protocols in these sets do not include constant amplitude or incrementally increasing tests which are considered standard in evaluating material model parameters in cyclic loading. 
Other noteworthy observations are that sets with smaller amplitudes generally lead to worst calibrations (c.f., e.g. set 19 vs. 28) and that the inclusion of a monotonic test is not necessarily essential to obtaining good parameter estimates as the results from set 45 imply. Furthermore, one can observe that testing using a random load protocol with non-zero mean strain (LP9) is important for the calibration procedure because it is present in almost all sets with a low number of protocols.

As to the nonuniqueness of the parameters, $\xi_{1}$ shows that the solutions are at least $40 \%$ different from each other. However, when one looks at what is the impact of that distance on the error function as evaluated by $\xi_{2}$, the effects are much more limited. Deciding on an acceptable level of $\xi_{2}$ is inherently linked to the acceptable difference in our error estimate from the base case. Although debatable, a value of $\xi_{2} \leq 5 \%$ seems small enough to be a suitable definition for consistency. Nevertheless, $\xi_{2}$ seems to indicate that most of the solutions obtained by the NTR algorithm are consistent with the base load protocol case, except for a few cases. Consider, for example, set 51 that has a relatively low $\bar{\varphi}$ value compared with set 2 which has the highest $\bar{\varphi}$. Here, set 51 is twice as far as set 2 in terms of $\xi_{2}$. This implies that set 51, which is conducted with four load protocols, is farther from the base case than a solution arrived at with only a simple monotonic test. Set 51, therefore, is a truly inconsistent result.

Solution consistency can be further analyzed by a thorough sensitivity assessment of the parameters. However, this would have to be performed at the solution point of each load protocol set, since they can have different sensitivities. This is recommended for further investigation, but preliminary findings show that the initial yield stress in the VC model has the most weight in the error of the model.

\section{Proposed material parameters for structural steels}

There are a few noteworthy comments to be made with respect to the material parameters. First and most conspicuous is the Young's modulus, which in Tables 2 and 5 is persistently lower than habitual values of $\approx 200-210 \mathrm{GPa}$ for mild steels. Interestingly, 
if only the monotonic test is used for the calibration the modulus is indeed close to the expected value. It is only with the addition of more load protocols, with higher accumulated plastic strains and the inclusion of load reversals, that on average the Young's modulus tends to drop.

Another puzzling result is that the estimation of initial yield stresses $\left(\sigma_{y, 0}\right)$ is also reliably lower than the yield plateau of the monotonic tests. This observation is associated with the fact that after the accumulated plastic strain passes the Lüders strain, the plateau phenomenon ceases to be present (Hall 1970). Upon load reversals, a smooth yielding phenomenon takes its place and starts at a lower yield level. Since most of the duration of the load protocols are spent in the smooth yielding range, what results from the optimization procedure is the incursion of a larger error at first yield in order to have smaller errors when reversals are made. It should be underscored that this is a limitation of the material model itself. Its impact could be felt in cases where the initial stress is paramount in determining structural behavior. Foremost among these are situations where geometric and material nonlinearities strongly interact (Hartloper et al. 2019). The discontinuous yielding phenomenon in mild steels makes it one of the most sensitive type of structural steels to this issue.

From Table 2, an important conclusion can be made as to the number of backstresses necessary to accurately capture material response. The gains in terms of $\bar{\varphi}$ are negligible after 2 backstresses are used. After this number, solution points can be consistent in the $\xi_{2}$ sense but do not bring any added value to the response. These results, therefore, suggest that 2 backstresses suffice in the description of the material response for earthquake situations.

Consistency in the solution points allows one to make meaningful qualitatively assertions about the parameter sets, and several examples can be produced from Table 5. For instance, one can readily identify materials of similar grades as illustrated by the similarities between the parameters of two different thickness plates of the same material grade, an S355J2+N. Their parameters are also close to those obtained for a nominally identical steel grade (S355J2) from wide-flange hot-rolled structural profiles (an HEB500 in the present 
case). Furthermore, it can also be stated that the higher the steel grade the lower the level of isotropic hardening, as shown by the decreasing values of $Q_{\infty}$. In fact, if one takes to evaluate the contribution of isotropic hardening in the total hardening behavior of the $\mathrm{VC}$ model at saturation, it can be seen that mild steels frequently present on the order of $25 \%$ of isotropic hardening whereas high strength steel (e.g. S690QL) is mostly kinematic. This observation is valid even between mild steels of different origin, such as the European S355 and the American A992 Gr. 50 steels. When viewed in the light of the classic Hall-Petch relationship that correlates increasing yield strength with decreasing grain size (Hall 1951, Petch 1953), the parameters obtained by this methodology seem to suggest that the amount of isotropic versus kinematic hardening in a material can also be linked to microstructural grounds. In this respect, however, it should also be pointed out that the manufacturing process (and, therefore, the material's grain size) need not be the only microstructural variable responsible for isotropic/kinematic ratio. Indeed, if one focuses on the Japanese steel BCR295, a lower grade steel (nominal yield stress of 295MPa) with presumably larger average grain size, a low level of isotropic hardening can be observed on the derived material parameters. Yet, the test specimens for this steel were sampled from the corner of a hollow square section (HSS), which implies that the material around this area had been cold-worked. Consequently, it can also be stated that previous work-hardening of the material can also have a significant impact on the proportion of isotropic to kinematic hardening.

On a separate note, comparisons between parameters found in literature and those proposed herein should be made with some care. There are two main sources of uncertainties in the determination of model parameters: (1) the inverse problem methodology and (2) inherent variations in the material properties from the manufacturing process. This paper addresses solely the first point. A lengthy comparison of the parameters proposed herein to those reported in literature would, therefore, be hard to parse out and is not attempted. It can be stated, nonetheless, that significant differences in terms of model performance can be found using literature parameters for the same material, but the source of that discrepancy 
still needs further investigation with larger datasets.

\section{CONCLUSION}

This paper presented an approach to solve an inverse problem on a classic metal plasticity, rate-independent, material model using a combined nonlinear isotropic and kinematic hardening according to the Voce and Chaboche rules (VC). The approach is based on the Newton Trust-Region methodology which was introduced and its implementation was discussed. An implementation of the procedure in the Python programming language is made freely available in de Castro e Sousa et al. (2019).

The methodology was applied to a wide variety of European, American and Japanese structural steels that are commonly used in steel construction worldwide and the resulting parameters are provided for reference.

The following overarching conclusions can be drawn for this work:

- Accurate derivative estimates and hessian preconditioning play a significant role in the convergence rate of the NTR method. Both Jacobi and SVD preconditioning work well in the present study;

- Solution points obtained with the NTR method for different starting points are close even in cases where the starting point is far away from the solution, e.g. $\bar{\varphi} \approx 100 \%$ where there is virtually no information on where the solution might lay, illustrating the robustness of the algorithm;

- Two backstresses in the Chaboche kinematic hardening rule seem sufficient to characterize material response for earthquake loading;

- Accuracy in cyclic loading is usually achieved at the noticeable expense of underestimating the initial yield stress with the Voce-Chaboche model;

- Nonuniqueness and consistency were analyzed and discussed. Their importance motivates the introduction of two metrics to quantify them. Load protocol sampling leads in large to consistent solutions using this approach; 
- The use of only three load protocols (LP2, LP3, and LP9) yield acceptable parameter estimates. These load protocols are different from the standard ones routinely used in material characterization in cyclic loading for seismic applications;

\section{DATA AVAILABILITY}

Some or all data, models, or code generated or used during the study are available in a repository or online in accordance with funder data retention policies. Some or all data, models, or code that support the findings of this study are available from the corresponding author upon reasonable request.

\section{ACKNOWLEDGMENTS}

The authors recognize and are grateful for the financial support of this study by the Nippon Steel Corporation, internal grants from École Polytechnique Fédérale de Lausanne (EPFL), as well as an exploratory grant from EPFL's School of Environmental, Architectural and Civil Engineering. The authors are also thankful to graduate student Alexander Hartloper for the data on S355J2 I-shape steel. Any opinions, findings, and conclusions or recommendations expressed in this paper are those of the authors and do not necessarily reflect the views of sponsors. 


\section{REFERENCES}

Adam, C. and Jäger, C. (2012). "Seismic collapse capacity of basic inelastic structures vulnerable to the P-delta effect." Earthquake Engineering 8 Structural Dynamics, 41, 775-793.

Andrade-Campos, A., Thuillier, S., Pilvin, P., and Teixeira-Dias, F. (2007). "On the determination of material parameters for internal variable thermoelastic-viscoplastic constitutive models." International Journal of Plasticity, 23(8), 1349-1379.

Araújo, M., Macedo, L., and Castro, J. M. (2017). "Evaluation of the rotation capacity limits of steel members defined in EC8-3." Journal of Constructional Steel Research, 135, 11-29.

Armstrong, P. and Fredrick, C. (1966). "A Mathematical Representation of the Multiaxial Bauschinger Effect." Report No. RD/B/N 731, Central Electricity Generating Board.

ASTM (2011). “ASTM E1049-85: Standard Practices for Cycle Counting in Fatigue Analysis." E1049 - 85, 85(Reapproved 2011), 1-10.

ASTM (2012). E606-E606M - 12 - Standard Test Method for Strain-Controlled Fatigue Testing. ASTM International, West Conshohocken, PA, USA.

ASTM (2013). E8/E8M - $13 a$ - Standard Test Methods for Tension Testing of Metallic Materials 1. ASTM International, West Conshohocken, PA, USA.

Baker, J. (2011). "Conditional Mean Spectrum: Tool for Ground-Motion Selection." Journal of Structural Engineering, 137(3), 322-331.

Bauschinger, J. (1874). Versuche uber die Festigkeit des Bessemer-Stahles von verschiedenem Kohlenstoffgehalt. Mitt. Mech.-Techn. Lab. K. Techn. Hochschule Munchen.

Bickel, B., Bächer, M., Otaduy, M. a., Matusik, W., Pfister, H., and Gross, M. (2009). "Capture and modeling of non-linear heterogeneous soft tissue." ACM Transactions on Graphics, 28(3), 1.

Bierlaire, M. (2015). Optimization: Principles and algorithms. EPFL Press, first edition.

Braconi, A., Finetto, M., Degee, H., Hausoul, N., Hoffmeister, B., Gündel, M., Karmanos, S., Pappa, P., Varelis, G., Rinaldi, V., and Obiala, R. (2013). "Optimising the seismic performance of steel and steel-concrete structures by standardising material quality control 
(OPUS)." Report No. 25893, European Commission, Luxembourg.

Brodtkorb, P. A. (2017). "numdifftools 0.9.20, < https://pypi.python.org/pypi/Numdifftools>. Bruhns, O. T. and Anding, D. (1999). "On the simultaneous estimation of model parameters used in constitutive laws for inelastic material behaviour." International Journal of Plasticity, 15, 1311-1340.

Chaboche, J., Dang Van, K., and Codier, G. (1979). "Modelization of the Strain Memory Effect on the Cyclic Hardening of 316 Stainless Steel." Structural mechanics in reactor technology - SMiRT 5, L11.

Chaboche, J. L. (2008). "A review of some plasticity and viscoplasticity constitutive theories." International Journal of Plasticity, 24(10), 1642-1693.

Conn, A. R., Gould, N. I., and Toint, P. L. (2000). Trust-Region Methods. Society for Industrial and Applied Mathematics and Mathematical Programming Society, Philadelphia, PA, United States of America.

Cooke, R. J. and Kanvinde, a. M. (2015). "Constitutive parameter calibration for structural steel: Non-uniqueness and loss of accuracy." Journal of Constructional Steel Research, $114,394-404$.

Dafalias, Y. F. (1986). "Bounding surface plasticity I: mathematical foundation and hypoelasticity." Journal of Engineering Mechanics, 112(9), 966-987.

Dafalias, Y. F., Kourousis, K. I., and Saridis, G. J. (2008). "Multiplicative AF kinematic hardening in plasticity." International Journal of Solids and Structures, 45(10), 2861-2880.

Dafalias, Y. F. and Popov, E. P. (1975). "A Model of Nonlinearly Hardening Materials for Complex Loading." Acta Mechanica, 21(3), 173-192.

de Castro e Sousa, A., Hartloper, A. R., and Lignos, D. G. (2019). "RESSPyLab version 1.0 - Python tools for structural steel research, <https://pypi.org/project/RESSPyLab/>.

Dehghani, M., Tremblay, R., and Leclerc, M. (2017). "Fatigue failure of 350WT steel under large-strain seismic loading at room and subfreezing temperatures." Construction and Building Materials, 145, 602-618. 
D'Errico, J. R. (2006). "Adaptive Robust Numerical Differentiation, <http://ch.mathworks.com/matlabcentral/fileexchange/13490-adaptive-robustnumerical-differentiation $>$.

Elkady, A. and Lignos, D. G. (2018). "Improved Seismic Design and Nonlinear Modeling Recommendations for Wide-Flange Steel Columns." Journal of Structural Engineering (United States), 144(9), 1-13.

Furukawa, T. and Yagawa, G. (1997). "Inelastic constitutive parameter identification using an evolutionary algorithm with continuous individuals." International Journal for Numerical Methods in Engineering, 40(6), 1071-1090.

Gendy, a. S. and Saleeb, a. F. (2000). "Nonlinear material parameter estimation for characterizing hyper elastic large strain models." Computational Mechanics, 25(1), 66-77.

Griewank, A. and Walther, A. (2008). Evaluating Derivatives: Principles and Techniques of Algorithmic Differentiation, Second Edition. Society for Industrial and Applied Mathematics and Mathematical Programming, Philadelphia, PA, United States of America.

Grigoriou, V. and Lignos, D. G. (2017). "Characterization of the cyclic hardening properties of european steels." Report No. (unpublished), EPFL - RESSLab.

Hall, E. O. (1951). "The deformation and aging of mild steel." Proceedings of the Physical Society of London B, 54, 747.

Hall, E. O. (1970). Yield Point Phenomena in Metals and Alloys. Plenum Press, New York, $<$ http://link.springer.com/content/pdf/10.1007/978-1-4684-1860-6.pdf>.

Hartloper, A. R., de Castro e Sousa, A., and Lignos, D. G. (2019). "Sensitivity of Simulated Steel Column Instabilities to Plasticity Model Assumptions." 12th Canadian conference on earthquake engineering, Chateau Frontenac, Quebec, Canada.

Hassan, T. and Kyriakides, S. (1992). "Ratcheting in cyclic plasticity, part I: Uniaxial behavior." International Journal of Plasticity, 8(1), 91-116.

Hill, R. (1950). The Mathematical Theory of Plasticity. Oxford University Press.

Hodge, P. G. (1956). "The theory of piecewise linear isotropic plasticity." Deformation and 
Flow of Solids/Verformung und Fliessen des Festkörpers, R. Grammel, ed., Vol. 267, Madrid, International Union of Theoretical and Applied Mechanics, 147-170.

Holzapfel, G. A., Gasser, T. C., and Ogden, R. A. Y. W. (2000). "A New Constitutive Framework for Arterial Wall Mechanics and a Comparative Study of Material Models." Journal of Elasticity and the physical science of solids, 61, 1-48.

ISO (2016). ISO 6892-1:2016 - Metallic Materials - Tensile testing - Part 1: Method of test at room temperature. International Organization for Standardization.

Kaufmann, E., Metrovich, B., and Pense, A. (2001). "Characterization of Cyclic Inelastic Strain Behavior On Properties of A572 Gr.50 and A913 Gr. 50 Rolled Sections." Report No. ATLSS No. 01-13, Lehigh University.

Kirkpatrick, S., Gelatt, C. D., and Vecchi, M. P. (1983). "Optimization by Simulated Annealing." Science, 220(4598), 671-680.

Koo, S., Han, J., Marimuthu, K. P., and Lee, H. (2019). "Determination of Chaboche combined hardening parameters with dual backstress for ratcheting evaluation of AISI 52100 bearing steel." International Journal of Fatigue, 122(January), 152-163.

Kostic, S. M. and Filippou, F. C. (2012). "Section discretization of fiber beam-column elements for cyclic inelastic response." Journal of Structural Engineering, 138(5), 592-601.

Krawinkler, H. (1996). "Cyclic Loading Histories for Seismic Experimentation on Structural Components." Earthquake Spectra, 12(1-12).

Krawinkler, H., Zohrei, M., Lashkari-Irvani, B., Cofie, N. G., and Hadidi-Tamjed, H. (1983). "Recommendations for Experimental Studies on the Seismic Behavior of Steel Components and Materials.." Report No. 61, Stanford University, John A. Blume Earthquake Engineering Center, Stanford, California, USA.

Lemaitre, J. and Desmorat, R. (2005). Engineering damage mechanics. Springer-Verlag.

Levenberg, K. (1944). "A method for the solution of certain non-linear problems in least squares." Quarterly of Applied Mathematics, 2(2), 164-168.

Lignos, D. G. and Krawinkler, H. (2013). "Development and utilization of structural com- 
ponent databases for performance-based earthquake engineering." Journal of Structural Engineering (United States), 139(8), 1382-1394.

Lignos, D. G., Krawinkler, H., and Whittaker, A. (2011). "Prediction and validation of sidesway collapse of two scale models of a 4-story steel moment frame." Earthquake Engineering \& Structural Dynamics, 40, 807-825.

Lubliner, J. (2008). Plasticity Theory. Dover Publications, Mineola, N.Y., U.S.A.

Ludwik, P. (1909). Elemente der technologischen Mechanik. Springer-Verlag, Berlin.

Marquadt, D. W. (1963). "An algorithm for least-squares estimation of nonlinear parameters." Journal of the Society for Industrial and Applied Mathematcs, 11(2).

McKenna, F. T. (1997). "Object-oriented finite element programming frameworks for analysis, algorithms and parallel computing." Ph.D. thesis, University of California, Berkeley, University of California, Berkeley.

Menegotto, M. and Pinto, P. E. (1973). "Method of analysis for cyclically loaded R.C. plane frames including changes in geometry and non-elastic behaviour of elements under combined normal force and bending." IABSE Symposium (Lisbon) on Resistance and Ultimate Deformability of Structures Acted on by Well Defined Loads, 15-22.

Miller, W. R. and Amin, H. S. (1975). "Low-cycle fatigue in welds." Experimental Mechanics, $15(6), 230-233$.

Mróz, Z. (1967). "On the description anisotropic workhardening." Journal of Mechanics and Physics of Solids, 15, 163-175.

Nabarro, F. R. N., Basinski, Z. S., and Holt, D. B. (1964). "The plasticity of pure single crystals." Advances in Physics, 13(50), 193-323.

Ohno, N. and Wang, J. D. (1993). "Kinematic hardening rules with critical state of dynamic recovery, part II: application to experiments of ratchetting behavior." International Journal of Plasticity, 9, 391-403.

Pal, S., Wathugala, G. W., and Kundu, S. (1996). "Calibration of a Constitutive Model Using Genetic Algorithms." Computers and Geotechnics, 19(4), 325-348. 
PEER (2010). "Guidelines for Performance-Based Seismic Design of Tall Buildings." Report No. 2010/05, Pacific Earthquake Engineering Research Center (PEER), Berkeley, California, U.S.A.

Petch, N. J. (1953). "The cleavage strength of polycrystals." Journal of the Iron and Steel Institute, 174, 25-28.

Prager, W. (1956). "A new method of analyzing stresses and strains in work hardening plastic solids." Journal of Applied Mechanics, 23, 493-496.

Rezaiee-Pajand, M. and Sinaie, S. (2009). "On the calibration of the Chaboche hardening model and a modified hardening rule for uniaxial ratcheting prediction." International Journal of Solids and Structures, 46(16), 3009-3017.

Simo, J. C. and Hughes, T. J. R. (1998). Computational inelasticity. Springer-Verlag, New York.

Smith, C., Kanvinde, a., and Deierlein, G. (2017). "Calibration of continuum cyclic constitutive models for structural steel using particle swarm optimization." Journal of Engineering Mechanics, 143(5), 1-10.

Spacone, E., Filippou, F. C., and Taucer, F. F. (1996). "Fibre beam-column model for non-linear analysis of R/C frames: Part 1. Formulation." Earthquake Engineering and Structural Dynamics, 25, 711-725.

Suzuki, Y. (2018). "Earthquake-induced collapse of steel moment resisting frames with conventional and high performance steel columns." Ph.D. thesis, McGill University, McGill University.

Suzuki, Y. and Lignos, D. G. (2015). "Large Scale Collapse Experiments of Wide Flange Steel Beam-Columns." Proceedings of the 8th International Conference on Behavior of Steel Structures in Seismic Areas (STESSA), Shanghai, China.

Suzuki, Y. and Lignos, D. G. (2019). "Development of collapse-consistent loading protocols for experimental testing of steel columns." Earthquake Engineering and Structural Dynamics, (accepted). 
Voce, E. (1948). "The relationship between stress and strain for homogenous deformation." Journal of Institute of Metals, 74, 537-562.

von Mises, R. (1928). "Mechanik der plastischen Formanderung von Kristallen." Zeitschrift fur Angewandte Mathematik und Mechanik, 8(3), 161-185.

Wales, D. (2003). Energy Lanscapes. Cambridge University Press.

Walter, S. F. and Lehmann, L. (2013). "Algorithmic differentiation in Python with AlgoPy." Journal of Computational Science, 4(5), 334-344.

Wilson, H. G. (1978). "Least Squares Versus Minimum Absolute Deviations Estimation in Linear Models." Decision Sciences, 9(2), 322-335.

Zareian, F., Krawinkler, H., Ibarra, L., and Lignos, D. (2010). "Basic concepts and performance measures in prediction of collapse of buildings under earthquake ground motions." Structural Design of Tall and Special Buildings, 19, 167-181.

Zareian, F. and Medina, R. A. (2010). "A practical method for proper modeling of structural damping in inelastic plane structural systems." Computers and Structures, 88, 45-53. 
NOTATION

The following symbols are used in this paper:

$$
\begin{aligned}
& \sigma=\text { uniaxial true stress }(\mathrm{Pa}) \text {; } \\
& \varepsilon=\text { uniaxial true strain; } \\
& \sigma_{e n g}=\text { uniaxial engineering stress }(\mathrm{Pa}) \text {; } \\
& \varepsilon_{\text {eng }}=\text { uniaxial engineering strain; } \\
& F=\text { actuator force through uniaxial specimen }(\mathrm{N}) \text {; } \\
& L=\text { gage length }(\mathrm{m}) \text {; } \\
& A=\text { uniaxial specimen's cross section area }\left(\mathrm{m}^{2}\right) \text {; } \\
& J_{2}=\text { second invariant of the deviatoric stress tensor; } \\
& \phi^{V M}=\text { Von Mises yield criterion; } \\
& E=\text { Young's modulus; } \\
& \sigma_{y}=\text { current yield stress }(\mathrm{Pa}) \text {; } \\
& \sigma_{y, 0}=\text { initial yield stress }(\mathrm{Pa}) \text {; } \\
& \Delta \sigma_{y}=\text { difference between current and initial yield stress }(\mathrm{Pa}) \text {; } \\
& Q_{\infty}=\text { isotropic differential stress at saturation }(\mathrm{Pa}) \text {; } \\
& b=\text { isotropic saturation rate parameter; } \\
& C_{k}=\text { kinematic stress parameter for the } k \text {-th backstress }(\mathrm{Pa}) \text {; } \\
& \gamma_{k}=\text { kinematic saturation rate parameter for the } k \text {-th backstress; } \\
& \boldsymbol{\theta}=\text { vector of all material parameters; } \\
& \dot{\varepsilon}_{e q}^{p}=\text { equivalent plastic strain; } \\
& \varepsilon^{*}=\text { error-metric strain; } \\
& \varphi=\text { error function; } \\
& \bar{\varphi}=\text { normalized error function; } \\
& m=\text { local quadratic approximation model of a function; } \\
& \nabla_{\mathbf{x}} f=\text { gradient of function } f \text { with respect to vector variable } \mathbf{x} \text {; } \\
& \nabla_{\mathbf{x x}} f=\text { hessian of function } f \text { with respect to vector variable } \mathbf{x} \text {; }
\end{aligned}
$$




$$
\begin{aligned}
\|\mathbf{x}\|_{l} & =\text {-norm of vector } \mathbf{x} ; \\
\mathbf{d} & =\text { step vector; } \\
\Delta & =\text { step magnitude limit; } \\
\rho & =\text { model fitness metric; } \\
\eta_{1} & =\text { lower bound on model fitness quality; } \\
\eta_{2} & =\text { upper bound on model fitness quality; } \\
\mathbf{w} & =\text { scaled step vector; } \\
\mathbf{S} & =\text { hessian preconditioner; } \\
\mathbf{H} & =\text { preconditioned hessian; } \\
\xi_{1} & =\text { uniqueness distance metric; } \\
\xi_{2} & =\text { consistency distance metric; }
\end{aligned}
$$




\section{List of Tables}

1 Summary of tensile properties for materials under study. . . . . . . . . . . . 41

2 Summary of NTR with SVD's performance and solution for different starting points and number of backstresses (S355J2+N - t=50mm) - 1/2 . . . . . 42

$3 \quad$ Summary of NTR with SVD's performance and solution for different starting points and number of backstresses (S355J2+N - t=50mm) - 2/2 . . . . . 43

4 Summary of normalized error metric for subset sampling. The analyses were conducted with 2 backstresses and the SVD preconditioning with starting point 1. . . . . . . . . . . . . . . . . . . . 44

$5 \quad$ Results from NTR-SVD for different materials with 2 backstresses . . . . . 45 
TABLE 1: Summary of tensile properties for materials under study.

\begin{tabular}{|c|c|c|c|c|c|}
\hline Material & $\begin{array}{l}E \\
(G P a)\end{array}$ & $\begin{array}{l}\sigma_{y} / \sigma_{y}^{\text {nominal }} \\
(M P a)\end{array}$ & $\begin{array}{l}\sigma_{0.2 p} \\
(M P a)\end{array}$ & $\begin{array}{l}\sigma_{u} \quad \mathbf{b} \\
(M P a)\end{array}$ & $\varepsilon_{u}{ }^{\mathbf{c}}$ \\
\hline $\mathrm{S} 355 \mathrm{~J} 2+\mathrm{N}\left(\right.$ Plt. $\left.^{\mathbf{a}}-50 \mathrm{~mm}\right)$ & 204 & $350 / 355$ & - & 537 & 0.171 \\
\hline $\mathrm{S} 355 \mathrm{~J} 2+\mathrm{N}\left(\right.$ Plt. $\left.^{\mathbf{a}}-25 \mathrm{~mm}\right)$ & 214 & $350 / 355$ & - & 543 & 0.176 \\
\hline S355J2 (Flange $\left.{ }^{\mathbf{e}}\right)$ & 219 & $325 / 355$ & - & $\mathrm{NA}^{\mathbf{d}}$ & $\mathrm{NA}^{\mathbf{d}}$ \\
\hline S355J2 $\left(\mathrm{Web}^{\mathbf{e}}\right)$ & 206 & $320 / 355$ & - & $\mathrm{NA}^{\mathbf{d}}$ & $\mathrm{NA}^{\mathrm{d}}$ \\
\hline S460NL (Plt. $\left.{ }^{\mathbf{a}}-25 \mathrm{~mm}\right)$ & 203 & $460 / 460$ & - & 643 & 0.166 \\
\hline S690QL (Plt. $\left.{ }^{\mathbf{a}}-25 \mathrm{~mm}\right)$ & 210 & $-/ 690$ & 712 & 797 & 0.078 \\
\hline A992 Gr.50 (Webf $)$ & 200 & $375 / 345$ & - & 504 & 0.183 \\
\hline A992 Gr.50 (Flange $\left.{ }^{\mathbf{f}}\right)$ & 204 & $360 / 345$ & - & 515 & 0.174 \\
\hline A500 Gr. B (HSS305x16) & 185 & $-/ 315$ & 373 & 439 & 0.184 \\
\hline BCP325 (Plt. $\left.{ }^{\mathrm{a}}-22 \mathrm{~mm}\right)$ & 212 & $380 / 325$ & - & 522 & 0.218 \\
\hline BCR295 (HSS350x22) & 187 & $-/ 295$ & 437 & 455 & 0.107 \\
\hline HYP400 (Plt. $\left.{ }^{\mathbf{a}}-27 \mathrm{~mm}\right)$ & 227 & $355 / 400$ & - & 535 & 0.209 \\
\hline
\end{tabular}

${ }^{\mathbf{a}}$ Plate; ${ }^{\mathbf{b}}$ ultimate engineering stress;

c the engineering strain at $\sigma_{u}$; ${ }^{\mathbf{d}}$ not available;

e specimens sampled from a HEB500 section;

f specimens sampled from a W14x82 section 
TABLE 2: Summary of NTR with SVD's performance and solution for different starting points and number of backstresses (S355J2+N - t=50mm) - 1/2

\begin{tabular}{|c|c|c|c|c|c|c|c|c|c|}
\hline S.P. ${ }^{a}$ & N.B. ${ }^{b}$ & $\varphi\left(M P a^{2}\right)$ & $\bar{\varphi}(\%)$ & $\|\varphi\|_{2}$ & It. $^{\mathrm{c}}$ & $E(G P a)$ & $\sigma_{y, 0}(M P a)$ & $Q_{\infty}(M P a)$ & $b$ \\
\hline 1 & 1 & 14925.68 & 8.21 & $3.1 \mathrm{e}-12$ & 34 & 177.54 & 296.62 & 123.26 & 7.17 \\
\hline 2 & 1 & 14925.68 & 8.21 & $2.5 \mathrm{e}-12$ & 39 & 177.54 & 296.62 & 123.26 & 7.17 \\
\hline 3 & 1 & 14925.68 & 8.21 & $3.3 \mathrm{e}-12$ & 41 & 177.54 & 296.62 & 123.26 & 7.17 \\
\hline 1 & $\overline{2}$ & $89 \overline{8} 0 . \overline{4} 8$ & $\overline{6} . \overline{3} \overline{7}$ & $5.8 \mathrm{e}-12$ & $\overline{4} \overline{8}$ & $\overline{1} \overline{8} 4 . \overline{9}$ & $27 \overline{0} . \overline{9} \bar{c}$ & $\overline{1} 0 \overline{2} . \overline{2}$ & $\overline{5} . \overline{9} \overline{7}$ \\
\hline 2 & 2 & 8980.48 & 6.37 & $4.6 \mathrm{e}-12$ & 48 & 184.98 & 270.96 & 107.22 & 5.97 \\
\hline 3 & 2 & 8980.48 & 6.37 & $2.3 \mathrm{e}-12$ & 53 & 184.98 & 270.96 & 107.22 & 5.97 \\
\hline$\overline{1}^{-}$ & 3 & $89 \overline{3} 1 . \overline{0}$ & $\overline{6} . \overline{3} \overline{5}$ & $5 . \overline{5}-\overline{1}-\overline{2}$ & $\overline{8} \overline{1}$ & $\overline{1} \overline{8} 5 . \overline{3}$ & $2 \overline{6} \overline{9} . \overline{2}$ & $\overline{1} \overline{0} .01^{-}$ & $\overline{6} . \overline{0} \overline{0}$ \\
\hline 2 & 3 & 8931.03 & 6.35 & $4.6 \mathrm{e}-12$ & 71 & 185.32 & 269.22 & 107.01 & 6.00 \\
\hline 3 & 3 & 8931.03 & 6.35 & $5.0 \mathrm{e}-12$ & 68 & 185.32 & 269.22 & 107.01 & 6.00 \\
\hline$\overline{1}-$ & $\overline{4}$ & $89 \overline{1} 1.03$ & $\overline{6} . \overline{3} \overline{5}$ & $\overline{6} . \overline{\mathrm{e}}-\overline{1} \overline{2}$ & $\overline{8} \overline{4}$ & 185.32 & $26 \overline{9} . \overline{2}$ & $\overline{107.01}$ & $\overline{6} . \overline{0} \overline{0}$ \\
\hline 2 & 4 & 8931.03 & 6.35 & $3.4 \mathrm{e}-12$ & 87 & 185.32 & 269.22 & 107.01 & 6.00 \\
\hline 3 & 4 & 8931.03 & 6.35 & $9.5 \mathrm{e}-12$ & 92 & 185.32 & 269.22 & 107.01 & 6.00 \\
\hline
\end{tabular}


TABLE 3: Summary of NTR with SVD's performance and solution for different starting points and number of backstresses (S355J2+N - t=50mm)- 2/2

\begin{tabular}{|c|c|c|c|c|c|c|c|c|c|}
\hline S.P. ${ }^{a}$ & N.B. ${ }^{b}$ & $C_{1}(M P a)$ & $\gamma_{1}$ & $C_{2}(M P a)$ & $\gamma_{2}$ & $C_{3}(M P a)$ & $\gamma_{3}$ & $C_{4}(M P a)$ & $\gamma_{4}$ \\
\hline 1 & 1 & 6501.83 & 27.90 & - & - & - & - & - & - \\
\hline 2 & 1 & 6501.83 & 27.90 & - & - & - & - & - & - \\
\hline 3 & 1 & 6501.83 & 27.90 & - & - & - & - & - & - \\
\hline 1 & $\overline{2}$ & 14327.30 & $11 \overline{5} .1 \overline{2}$ & $\overline{1} \overline{7} \overline{1} .0 \overline{6}$ & $\overline{7} . \overline{5} \overline{6}$ & 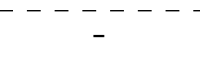 & - & $=$ & $=$ \\
\hline 2 & 2 & 14327.30 & 115.12 & 1771.06 & 7.56 & - & - & - & - \\
\hline 3 & 2 & 14327.30 & 115.12 & 1771.06 & 7.56 & - & - & - & - \\
\hline 1 & $\overline{3}$ & $\overline{1} \overline{2} \overline{6} 59.4 \overline{3}$ & $150.9 \overline{4}$ & $\overline{3} 2 \overline{2} \overline{0} . \overline{7}$ & $5 \overline{1.5} \overline{5}$ & $\overline{1} \overline{3} \overline{5} . \overline{22}$ & $5 . \overline{5} 4$ & $=$ & - \\
\hline 2 & 3 & 12659.43 & 150.94 & 3226.07 & 51.55 & 1345.22 & 5.54 & - & - \\
\hline 3 & 3 & 12659.43 & 150.94 & 3226.07 & 51.55 & 1345.22 & 5.54 & - & - \\
\hline 1 & 4 & $\overline{7} \overline{9} \overline{0} \overline{0} \overline{7} \overline{8}$ & 150.94 & $\overline{5} 3 \overline{8} .6 \overline{6}$ & $\overline{150.94}$ & $\overline{3} \overline{2} \overline{6} \overline{0} . \overline{-}$ & $5 \overline{1} . \overline{5}$ & $\overline{1} 3 \overline{4} . \overline{2} \overline{2}$ & $\overline{5} . \overline{5}$ \\
\hline 2 & 4 & 6595.56 & 150.94 & 6063.88 & 150.94 & 3226.07 & 51.55 & 1345.22 & 5.54 \\
\hline 3 & 4 & 6359.00 & 150.94 & 6300.42 & 150.94 & 3226.07 & 51.55 & 1345.22 & 5.54 \\
\hline
\end{tabular}


TABLE 4: Summary of normalized error metric for subset sampling. The analyses were conducted with 2 backstresses and the SVD preconditioning with starting point 1.

\begin{tabular}{|c|c|c|c|c|c|c|c|c|c|c|c|}
\hline Set & $\# \mathrm{LP}^{\mathrm{a}}$ & $\mathrm{LP}^{\mathrm{b}}$ & $\bar{\varphi}$ & $\xi_{1}$ & $\xi_{2}$ & Set & $\# \mathrm{LP}^{\mathrm{a}}$ & $\mathrm{LP}^{\mathbf{b}}$ & $\bar{\varphi}$ & $\xi_{1}$ & $\xi_{2}$ \\
\hline \multirow{3}{*}{1} & \multirow{3}{*}{10} & $\begin{array}{l}1,2,3 \\
4,5,6\end{array}$ & \multirow{3}{*}{6.37} & \multirow{3}{*}{0.0} & \multirow{3}{*}{0.00} & 36 & 6 & $\begin{array}{l}1,3,6 \\
7,9,10\end{array}$ & 6.87 & 52.0 & 4.30 \\
\hline & & $7,8,9$ & & & & 19 & 4 & $1,3,5,9$ & 6.88 & 139.0 & 5.41 \\
\hline & & $\begin{array}{l}10 \\
1,2,4,\end{array}$ & & & & 40 & 5 & $\begin{array}{l}1,2,6, \\
8,10\end{array}$ & 6.90 & 71.0 & 4.30 \\
\hline \multirow[t]{2}{*}{44} & \multirow[t]{2}{*}{8} & $5,6,7$ & \multirow[t]{2}{*}{6.46} & \multirow[t]{2}{*}{42.0} & \multirow[t]{2}{*}{1.62} & 16 & 4 & $1,5,6,7$ & 6.90 & 88.0 & 4.88 \\
\hline & & 9,10 & & & & 31 & 3 & $1,3,10$ & 6.95 & 76.0 & 4.98 \\
\hline 26 & 5 & $\begin{array}{l}1,5,6 \\
8,9\end{array}$ & 6.53 & 58.0 & 2.62 & 32 & 4 & $\begin{array}{l}1,3,5 \\
10\end{array}$ & 6.95 & 152.0 & 7.00 \\
\hline 45 & 3 & $2,3,9$ & 6.55 & 83.0 & 3.43 & 51 & 4 & $2,4,6,9$ & 7.02 & 394.0 & 79.79 \\
\hline 34 & 6 & $\begin{array}{l}1,3,5 \\
7,9,10\end{array}$ & 6.55 & 70.0 & 2.72 & 35 & & $\begin{array}{l}1,3, \\
7,10\end{array}$ & 7.04 & 50.0 & 4.69 \\
\hline 18 & 3 & $1,3,9$ & 6.55 & 92.0 & 3.25 & 29 & 5 & $1,2,4$ & & & \\
\hline 42 & 6 & $\begin{array}{l}1,5,6 \\
8,9,10\end{array}$ & 6.57 & 57.0 & 3.14 & 13 & 4 & $\begin{array}{l}6,9 \\
1,3,5,7\end{array}$ & 7.00 & 105.0 & 08.18 \\
\hline \multirow{2}{*}{25} & 5 & $1,5,6$ & 6.57 & 640 & 279 & 9 & 2 & 1,6 & 7.16 & 124.0 & 11.92 \\
\hline & $J$ & 7,9 & 0.01 & 04.0 & 2.19 & 14 & 4 & $1,3,5,8$ & 7.23 & 170.0 & 8.27 \\
\hline 20 & 5 & $1,3,5$ & 658 & 700 & 283 & 8 & 3 & $1,3,5$ & 7.28 & 197.0 & 9.64 \\
\hline 20 & $J$ & 7,9 & 0.08 & 19.0 & 2.03 & 12 & 4 & $1,2,4,6$ & 7.33 & 395.0 & 79.23 \\
\hline 43 & 6 & $1,4,6$ & 661 & 690 & 522 & 6 & 2 & 1,5 & 7.33 & 182.0 & 7.75 \\
\hline & & $8,9,10$ & & & & 27 & 3 & $1,2,9$ & 7.38 & 127.0 & 5.69 \\
\hline 49 & 4 & $2,3,6$ & 6.65 & 57.0 & 3.97 & 17 & 2 & 1,9 & 7.54 & 126.0 & 5.44 \\
\hline 49 & 4 & & $0.0 \mathrm{~J}$ & 31.0 & 3.91 & 10 & 2 & 1,7 & 7.71 & 77.0 & 5.96 \\
\hline 21 & 4 & $1,3,6$ & 6.65 & 57.0 & 3.69 & 11 & 2 & 1,8 & 7.74 & 129.0 & 7.79 \\
\hline 21 & 4 & 9 & 0.00 & 31.0 & & 38 & 3 & $1,2,10$ & 7.80 & 224.0 & 9.49 \\
\hline 33 & 5 & $1,3,5$ & 6.70 & 94.0 & 3.88 & 37 & 2 & 1,10 & 7.90 & 219.0 & 9.67 \\
\hline & & 7,10 & 0.10 & 94.0 & 0.00 & 30 & 2 & 1,10 & 7.90 & 219.0 & 9.67 \\
\hline 23 & 5 & $\begin{array}{l}1,4,6, \\
7,9\end{array}$ & 6.74 & 105.0 & 10.89 & 39 & 4 & $\begin{array}{l}1,2,4, \\
10\end{array}$ & 8.31 & 142.0 & 7.76 \\
\hline 24 & 5 & $1,4,6$, & 6.75 & 112.0 & 14.78 & 3 & 2 & 1,2 & 8.45 & 113.0 & 9.82 \\
\hline 24 & $J$ & 8,9 & 0.10 & 112.0 & 14.10 & 47 & 3 & $2,4,9$ & 9.04 & 258.0 & 14.47 \\
\hline 41 & 6 & $1,2,6$ & 6.80 & 73.0 & 4.22 & 28 & 4 & $1,2,4,9$ & 9.12 & 253.0 & 13.32 \\
\hline 41 & 0 & $8,9,10$ & 0.00 & 10.0 & 4.22 & 4 & 2 & 1,3 & 9.32 & 164.0 & 14.21 \\
\hline 22 & 5 & $1,3,6$, & 6.81 & 48.0 & 3.99 & 7 & 3 & $1,2,4$ & 10.65 & 290.0 & 15.84 \\
\hline & & 7,9 & & & & 5 & 2 & 1,4 & 10.99 & 263.0 & 13.19 \\
\hline 15 & 4 & $1,5,6$ & 6.83 & 82.0 & 4.38 & 48 & 3 & $4,6,9$ & 13.62 & 450.0 & 72.53 \\
\hline & & & & & & 2 & 1 & 1 & 21.15 & 324.0 & 38.92 \\
\hline 46 & 3 & $2,6,9$ & 6.87 & 99.0 & 9.78 & & & mode & $a g d n r$ & & mnles. \\
\hline
\end{tabular}


TABLE 5: Results from NTR-SVD for different materials with 2 backstresses

\begin{tabular}{|c|c|c|c|c|c|c|c|c|c|c|}
\hline Material & $\bar{\varphi}(\%)$ & $\mathrm{LP}^{\mathrm{a}}$ & $\begin{array}{l}E \\
(G P a)\end{array}$ & $\begin{array}{l}\sigma_{y, 0} \\
(M P a)\end{array}$ & $\begin{array}{l}Q_{\infty} \\
(M P a)\end{array}$ & $b$ & $\begin{array}{l}C_{1} \\
(M P a)\end{array}$ & $\gamma_{1}$ & $\begin{array}{l}C_{2} \\
(M P a)\end{array}$ & $\gamma_{2}$ \\
\hline $\begin{array}{l}\text { S355J2+N } \\
\left(\text { Plt. }^{\mathbf{b}} \quad-\right. \\
50 \mathrm{~mm})\end{array}$ & 6.37 & 1 to 10 & 185 & 271 & 107 & 5.97 & 14330 & 115 & 1771.06 & 7.56 \\
\hline $\begin{array}{l}\text { S355J2+N } \\
\text { (Plt. }{ }^{\mathbf{b}} \quad- \\
25 \mathrm{~mm})\end{array}$ & 6.70 & 1 to 10 & 192 & 265 & 104 & 11.63 & 13000 & 100 & 1560.41 & 7.35 \\
\hline $\begin{array}{l}\text { S355J2 } \\
\left(\text { Flange }^{d}\right)\end{array}$ & 4.63 & $\begin{array}{l}1,2,5 \\
6,9\end{array}$ & 192 & 246 & 120 & 8.67 & 14020 & 205 & 1247.05 & 4.45 \\
\hline $\begin{array}{l}\text { S355J2 } \\
\left(\text { Web }^{\mathbf{d}}\right)\end{array}$ & 6.16 & $1-3,5-9$ & 198 & 252 & 118 & 10.85 & 17876 & 236 & 2582.61 & 24.01 \\
\hline $\begin{array}{l}\text { S460NL } \\
\left(\text { Plt. }^{\mathbf{b}} \quad-\right. \\
25 \mathrm{~mm})\end{array}$ & 6.32 & $\begin{array}{l}1,2,4,5 \\
6,7,9,10\end{array}$ & 186 & 359 & 68 & 10.01 & 14202 & 104 & 2259.11 & 8.01 \\
\hline $\begin{array}{l}\text { S690QL } \\
\text { (Plt. }^{\text {b }} \\
25 \mathrm{~mm})\end{array}$ & 7.95 & $\begin{array}{l}1,2,4,5 \\
6,7,9,10\end{array}$ & 184 & 603 & 0.48 & 0.54 & 15938 & 78.8 & 988.77 & 6.57 \\
\hline $\begin{array}{l}\text { A } 992 \\
\text { Gr.50 } \\
\left(\text { Web }^{c}\right)\end{array}$ & 7.01 & $1,5,6,8,9$ & 183 & 339.18 & 78 & 9.29 & 8716 & 118 & 1182 & 5.22 \\
\hline $\begin{array}{l}\text { A992 } \\
\text { Gr.50 } \\
\left(\text { Flange }^{c}\right)\end{array}$ & 7.31 & $1,5,6,8,9$ & 180 & 318.47 & 101 & 8.00 & 11608 & 145.22 & 1026 & 4.68 \\
\hline $\begin{array}{l}\text { A500 } \\
\text { Gr. B } \\
(\text { HSS305x16) }\end{array}$ & 6.86 & $1,5,6,8,9$ & 171 & 301.26 & 130 & 82.2 & 9752 & 151.55 & 1613 & 8.21 \\
\hline $\begin{array}{l}\text { BCP325 } \\
\text { (Plt. }^{\mathbf{b}} \\
22 \mathrm{~mm})\end{array}$ & 5.05 & $1,5,6,8,9$ & 178 & 306.09 & 94 & 5.81 & 11613 & 122.00 & 1744 & 8.29 \\
\hline $\begin{array}{l}\text { BCR295 } \\
(\text { HSS350x22) }\end{array}$ & 8.38 & $1,5,6,8,9$ & 173 & 346.42 & 0.56 & 0.60 & 9746 & 197.67 & 1852 & 12.84 \\
\hline $\begin{array}{l}\text { HYP400 } \\
\text { (Plt. }^{\mathbf{b}} \quad- \\
27 \mathrm{~mm})\end{array}$ & 5.31 & $1,5,6,8,9$ & 189 & 376.22 & 29 & 6.41 & 13712 & 139.70 & 1147 & 4.59 \\
\hline
\end{tabular}

a load protocols used in calibration; ${ }^{\mathbf{b}}$ Plate; c specimens sampled from a W14x82 section; ${ }^{\mathbf{d}}$ specimens sampled from an HEB500 section 


\section{List of Figures}

1 Graphical representation of the error function $\varphi$ as the square of the shaded

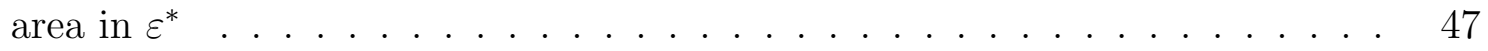

2 Level curves of function $f$, Taylor approximation $m$, and illustration of step size according to different $l$-norms . . . . . . . . . . . . . . . . . . . 48

$3 \quad$ Function and model values along the search direction in Fig. 2a and 2b . . . 49

4 Loading protocols under investigation; the loading excursion axis is schematic 50

5 Performance of the NTR algorithm with Jacobian preconditioning with respect to the use of numerical vs. algorithmic differentiation . . . . . . . . . . 51

6 Evolution of the gradient for a two backstress model for NTR with: no preconditioning(NTR), Jacobi (NTR_J) and SVD preconditioning(NTR_SVD) . 52

7 Two backstress model parameter evolution for the preconditioned Newton Trust-Region algorithm at three different starting points - $(\mathrm{J})$ Jacobi preconditioning; (SVD) Singular value decomposition preconditioning. . . . . . . . 53

8 Comparison of test results and the combined Voce and Chaboche model with two backstresses with parameters estimated with NTR $(J)$ for S355J2+N $(\mathrm{t}=50 \mathrm{~mm})$ steel . . . . . . . . . . . . . . . . . . . 54

8 (Cont.) Comparison of test results and the combined Voce and Chaboche model with two backstresses estimated with NTR $(\mathrm{J})$ for S355J2+N ( $\mathrm{t}=50 \mathrm{~mm})$

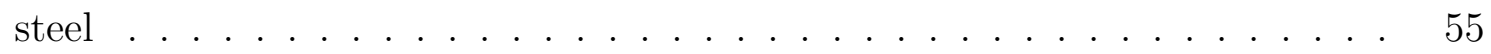

9 Evolution of the minimum eigenvalue sign of the Hessians in the NTR with SVD preconditioning method for different starting points and number of back-

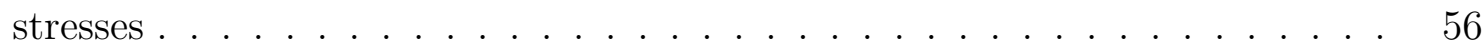



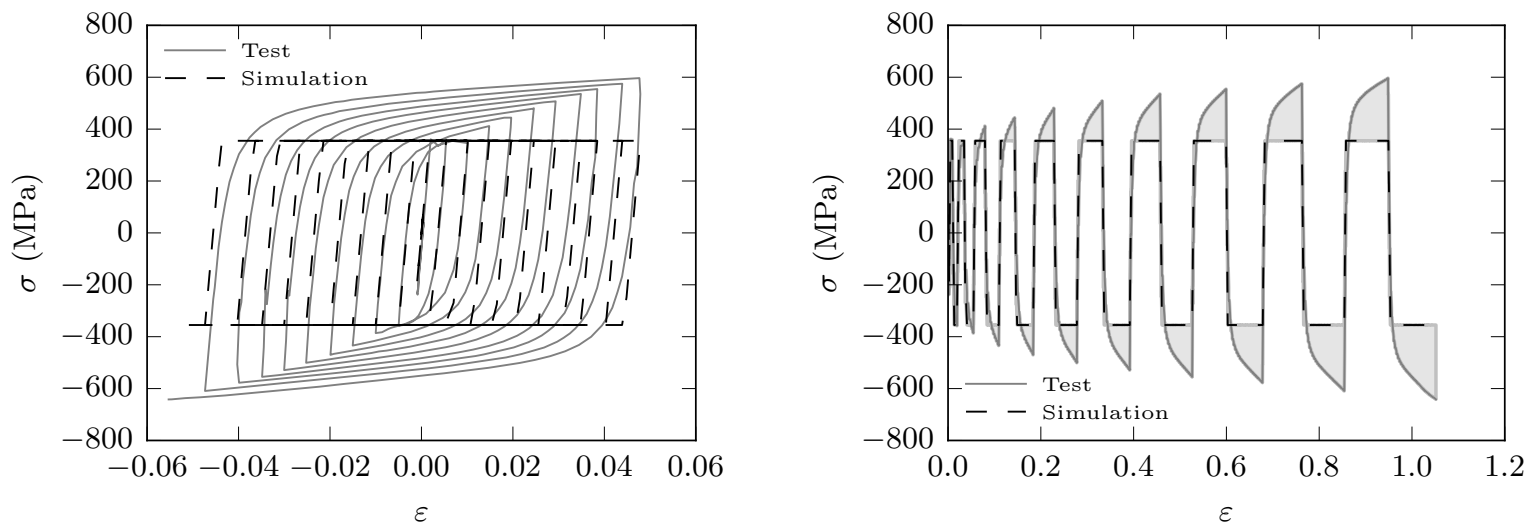

(a) Stress-strain relation of an example load pro- (b) Unpacked load protocol and shaded area as a tocol and corresponding estimate of an elastic- metric for the error in the elastic-perfectly plasperfectly plastic model tic model

FIG. 1: Graphical representation of the error function $\varphi$ as the square of the shaded area in $\varepsilon^{*}$ 


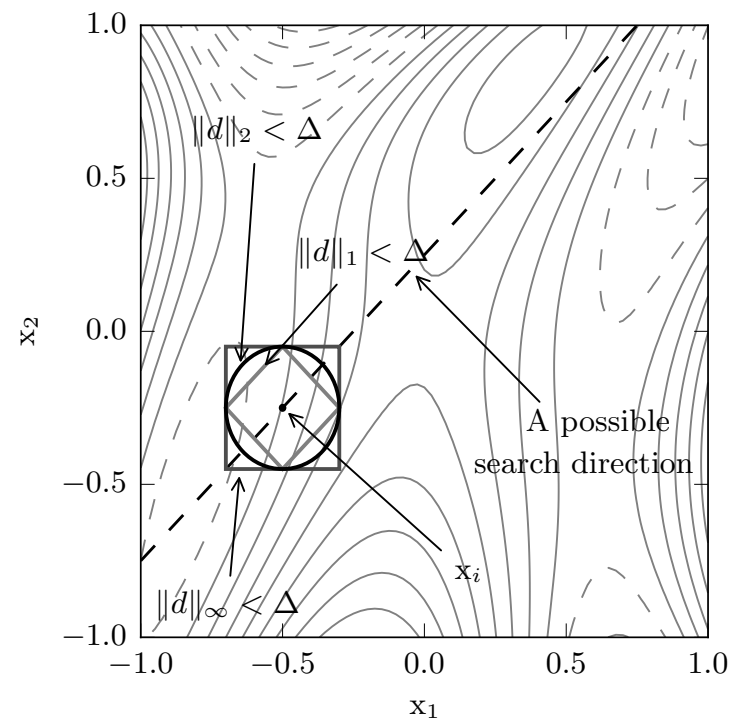

(a) Function $f$

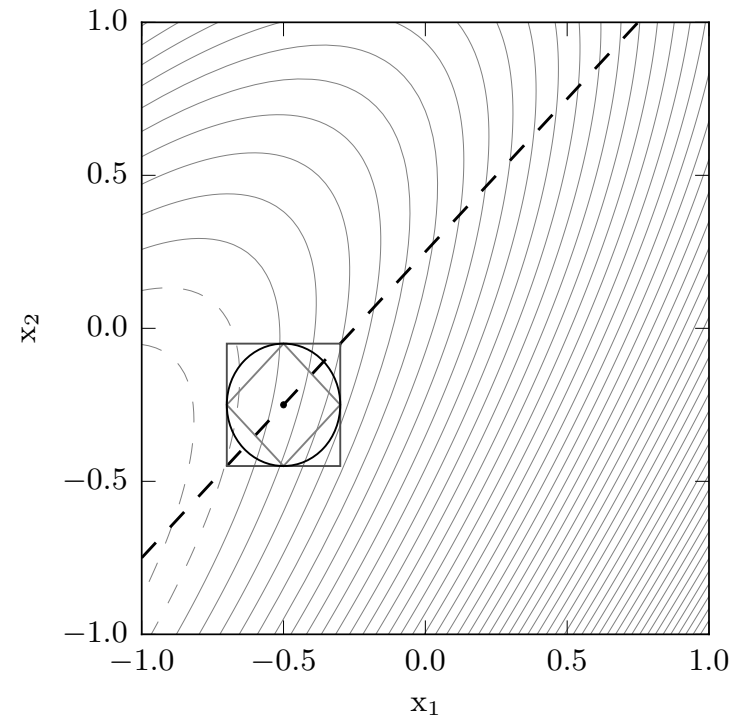

(b) Taylor approximation $m$ centered on $\boldsymbol{x}_{i}$

FIG. 2: Level curves of function $f$, Taylor approximation $m$, and illustration of step size according to different $l$-norms 


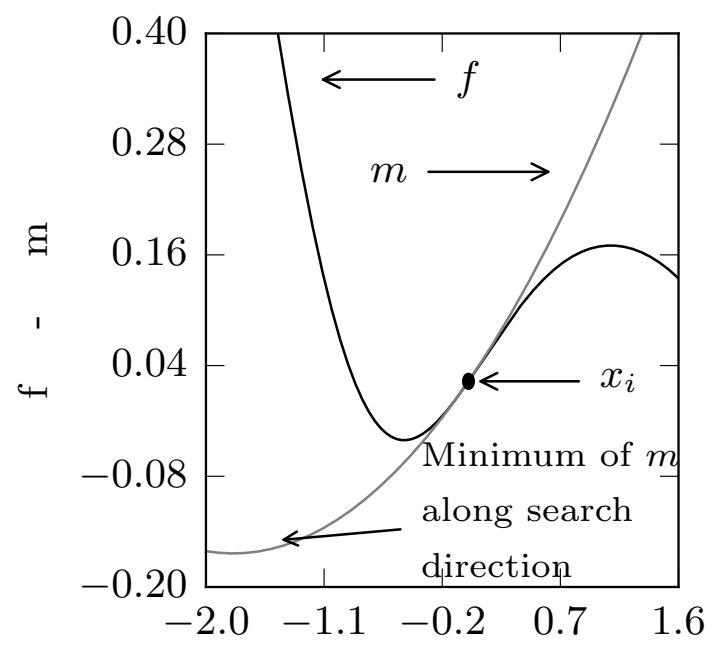

Length in the search direction

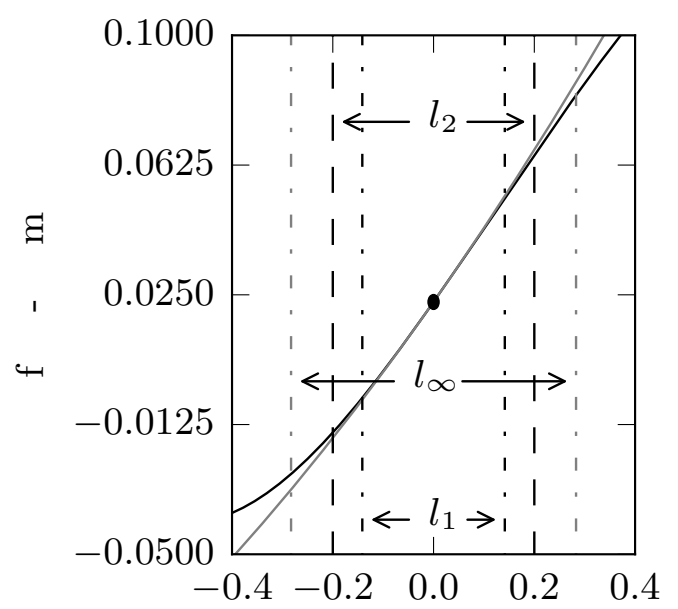

Length in the search direction

(b) Step size restrictions according to different

(a) Mismatch between $f$ and $m$ away from $\boldsymbol{x}_{i} \quad l$-norms

FIG. 3: Function and model values along the search direction in Fig. 2a and 2b 


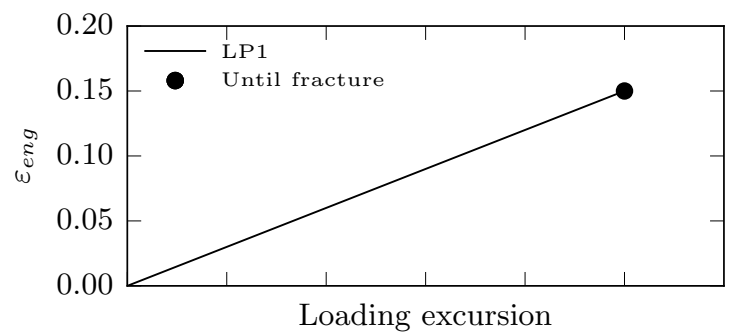

(a) Loading protocol \# 1 - monotonic tensile test

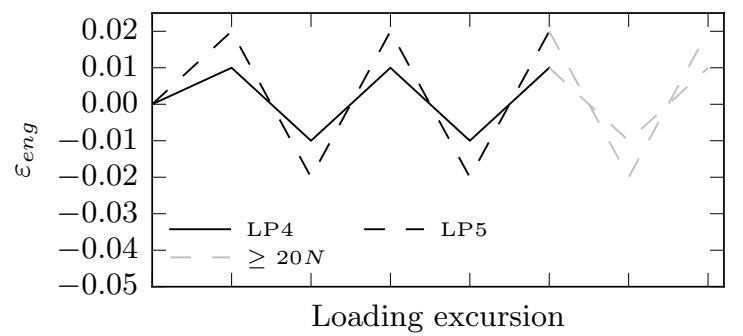

(c) Loading protocol \# 4 \& 5 - constant amplitude in ranges of $2 \%$ and $4 \%$ with zero mean strain, respectively

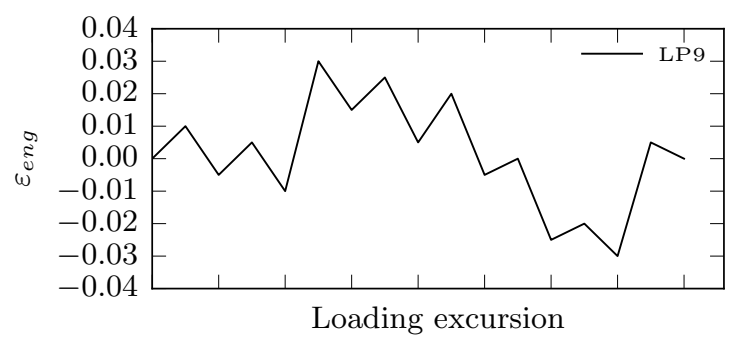

(e) Loading protocol \# 9 - variable amplitude

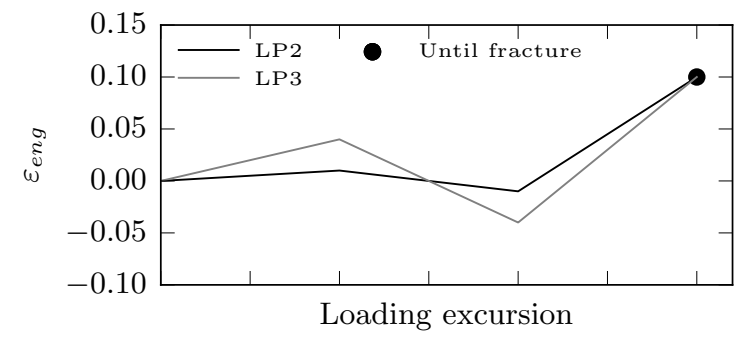

(b) Loading protocol \# 2 \& 3 - One cycle at $1 \%$ and $4 \%$ to fracture, respectively

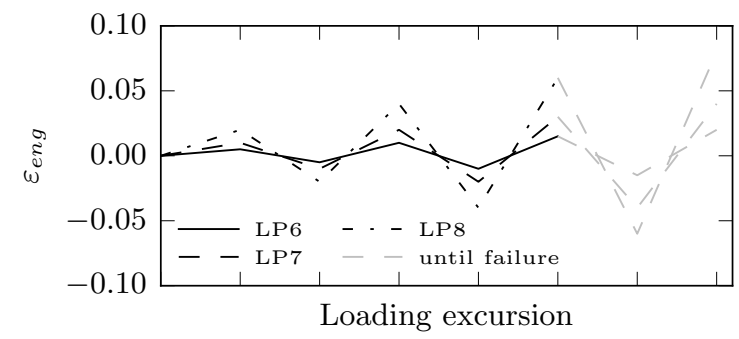

(d) Loading protocol \# 6, 7 \& 8 - incrementally increasing ranges of $1 \%, 2 \%$ and $4 \%$, respectively, until specimen failure

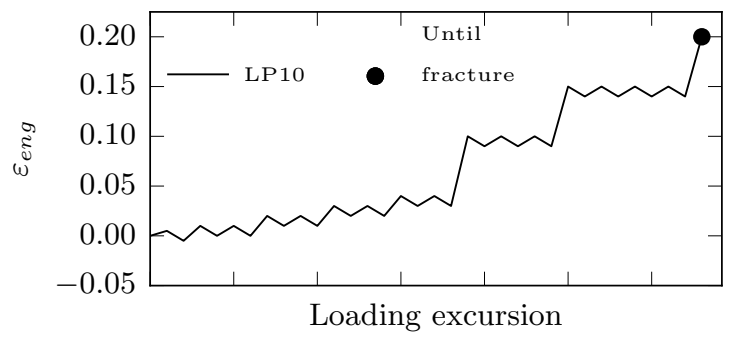

(f) Loading protocol \# 10 - asymmetric

FIG. 4: Loading protocols under investigation; the loading excursion axis is schematic 


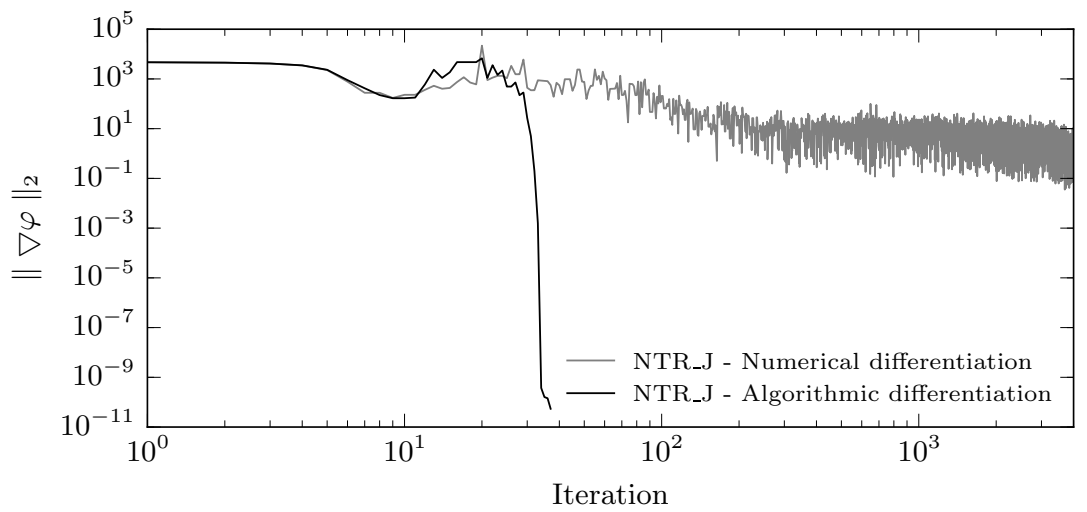

FIG. 5: Performance of the NTR algorithm with Jacobian preconditioning with respect to the use of numerical vs. algorithmic differentiation 


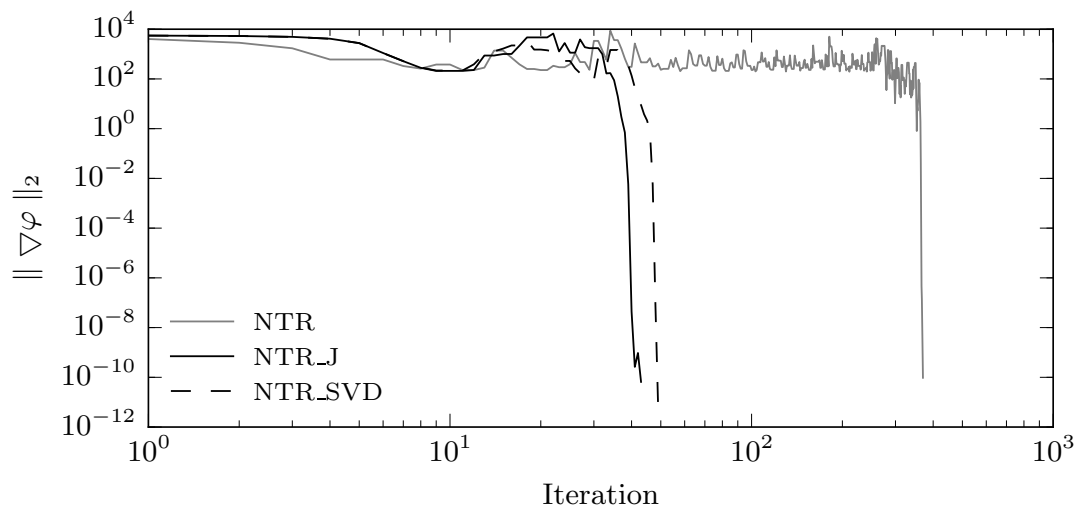

FIG. 6: Evolution of the gradient for a two backstress model for NTR with: no preconditioning(NTR), Jacobi (NTR_J) and SVD preconditioning(NTR_SVD) 


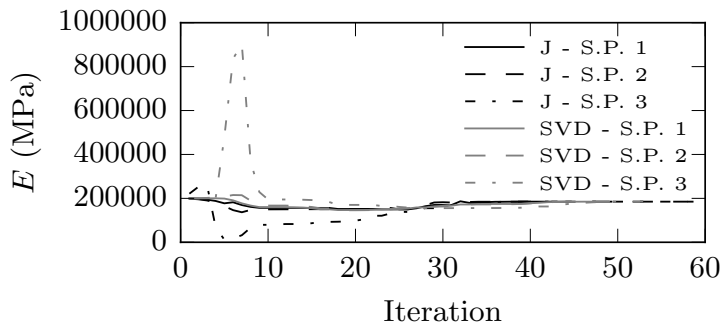

(a) Elastic modulus

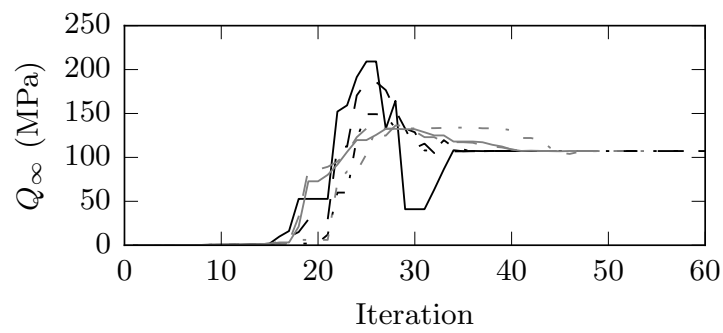

(c) Isotropic hardening parameter

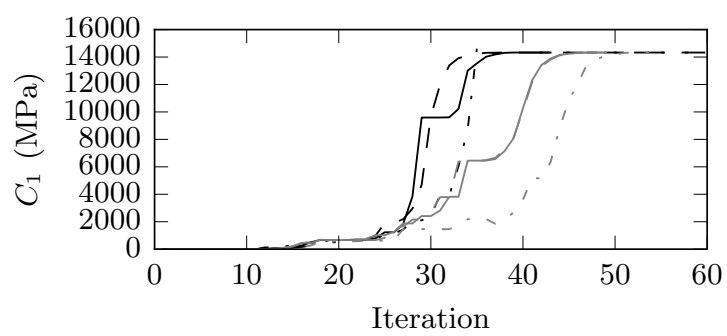

(e) $1^{\text {st }}$ Kinematic hardening parameter - 1st (f) $1^{\text {st }}$ Kinematic decay parameter - 1st backbacktress

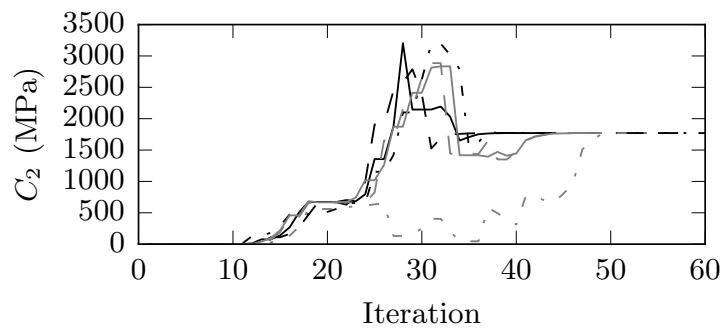

(g) $1^{s t}$ Kinematic hardening parameter - 2nd (h) $1^{s t}$ Kinematic decay parameter- 2nd backbackstress

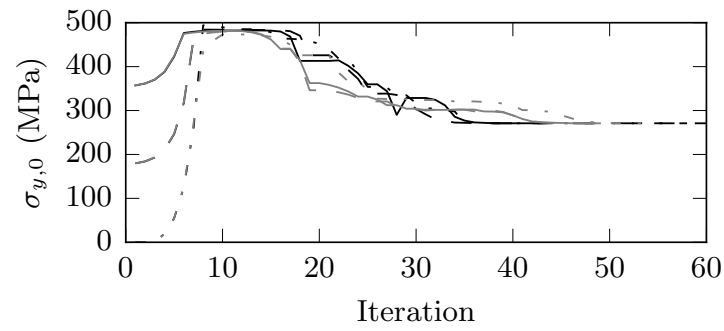

(b) Initial yield stress

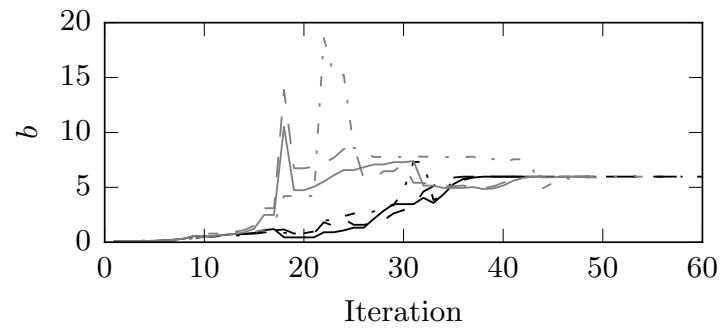

(d) Isotropic decay parameter

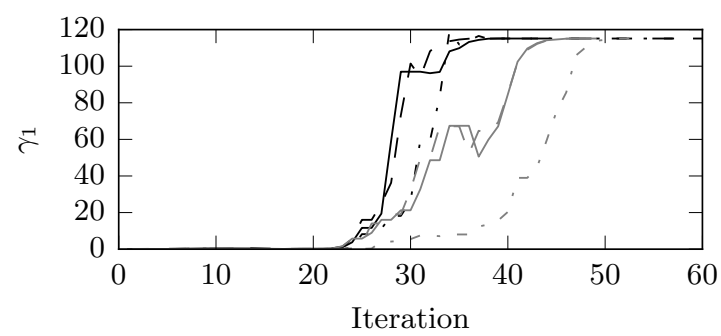
stress

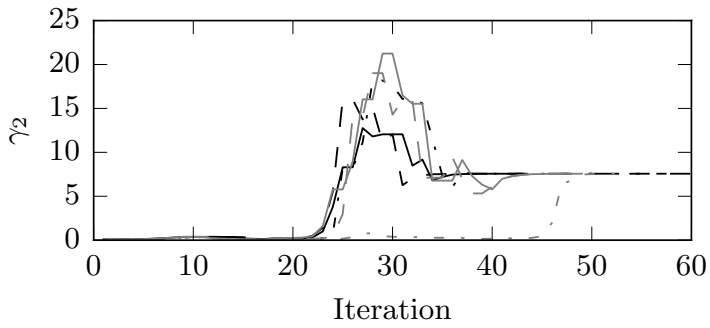

FIG. 7: Two backstress model parameter evolution for the preconditioned Newton TrustRegion algorithm at three different starting points - (J) Jacobi preconditioning; (SVD) Singular value decomposition preconditioning. 


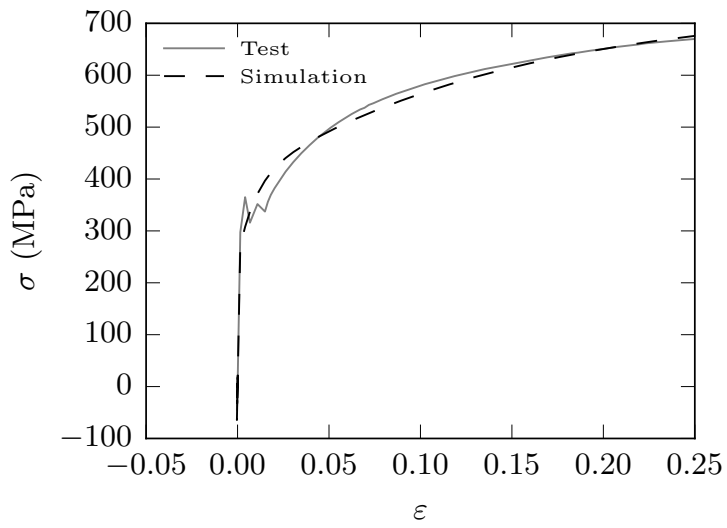

(a) Load protocol \# 1

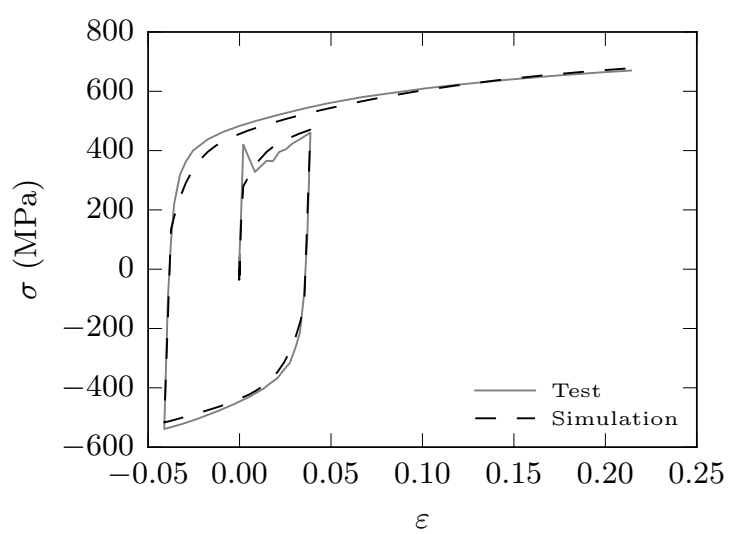

(c) Load protocol \# 3

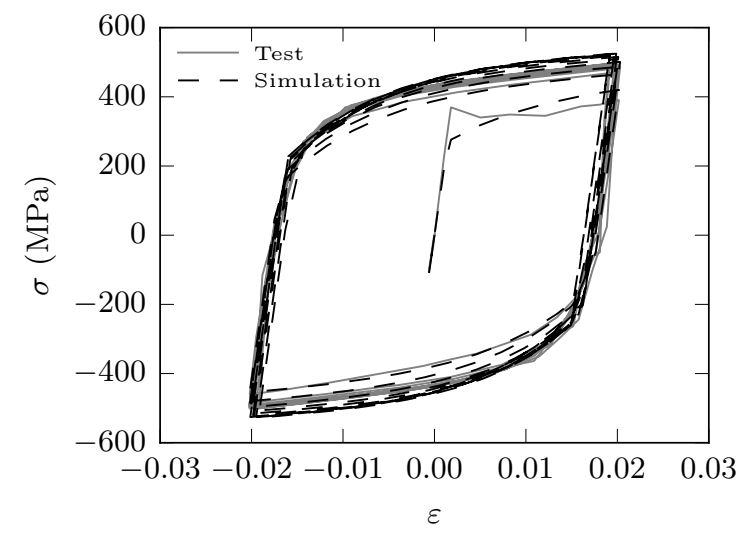

(e) Load protocol \# 5

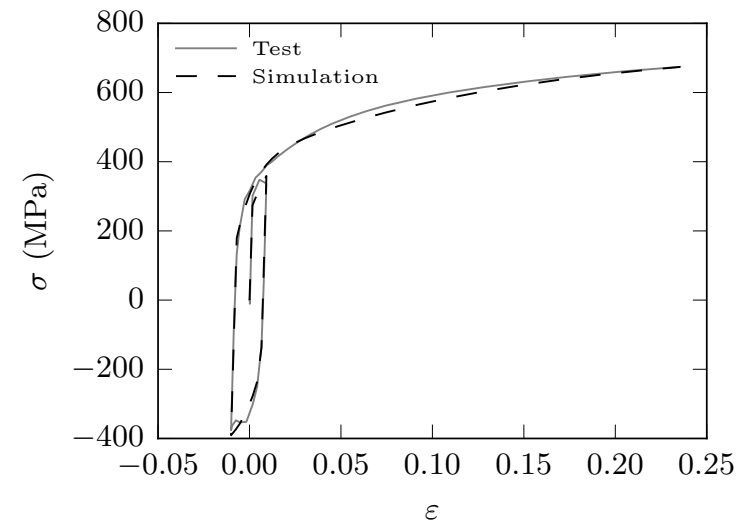

(b) Load protocol \# 2

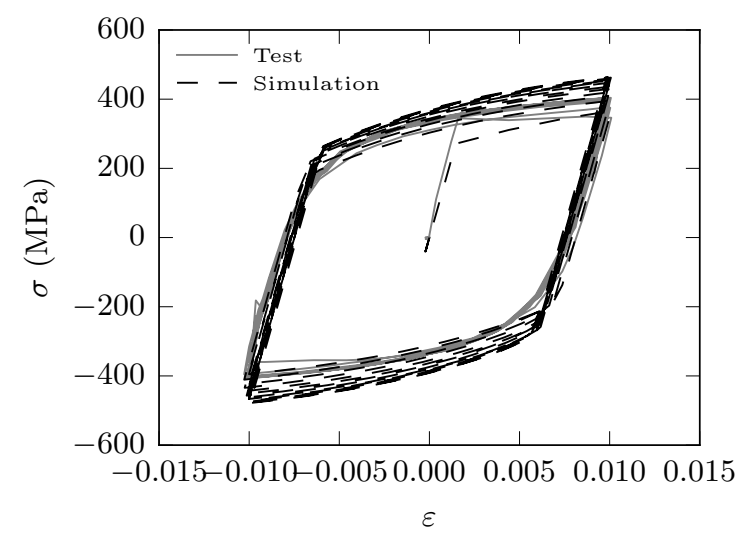

(d) Load protocol \# 4

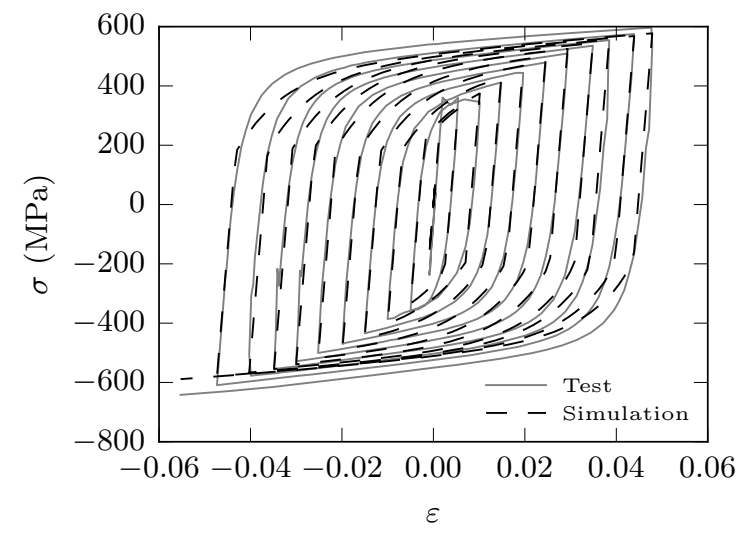

(f) Load protocol \# 6

FIG. 8: Comparison of test results and the combined Voce and Chaboche model with two backstresses with parameters estimated with NTR $(\mathrm{J})$ for S355J2+N ( $\mathrm{t}=50 \mathrm{~mm})$ steel 


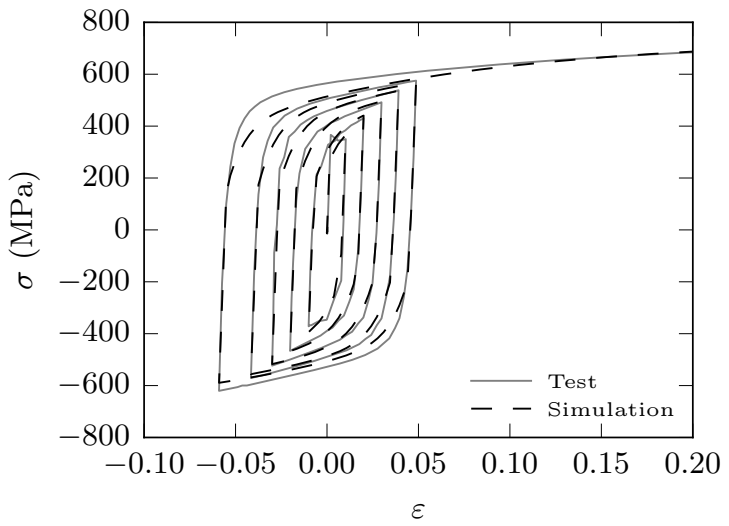

(g) Load protocol \# 7

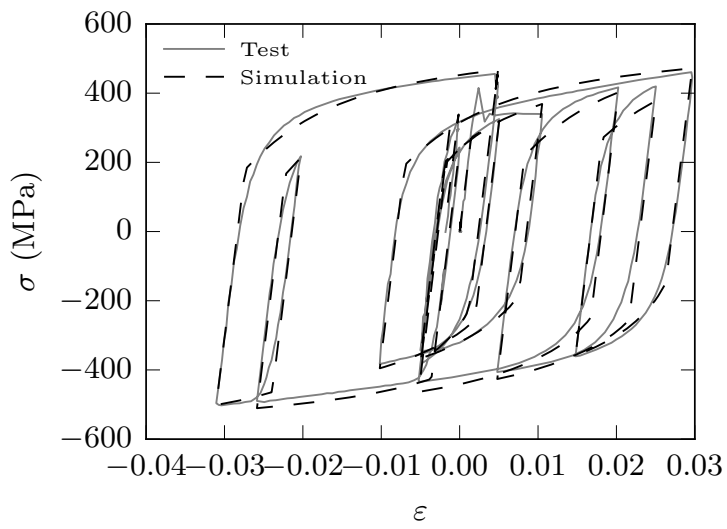

(i) Load protocol \# 9

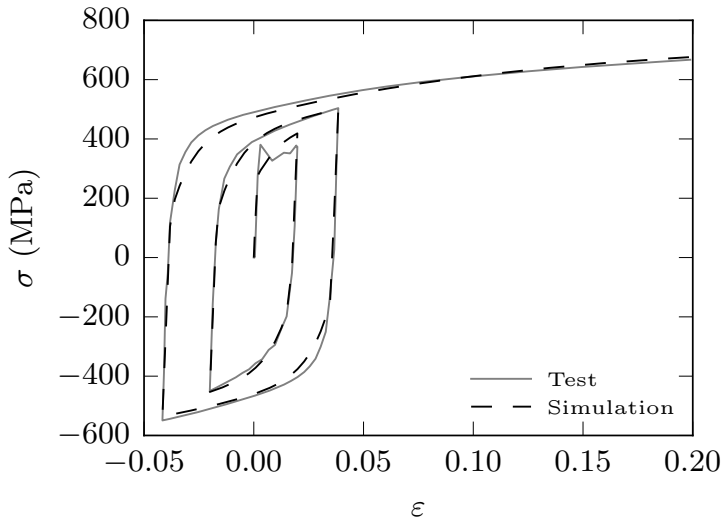

(h) Load protocol \# 8

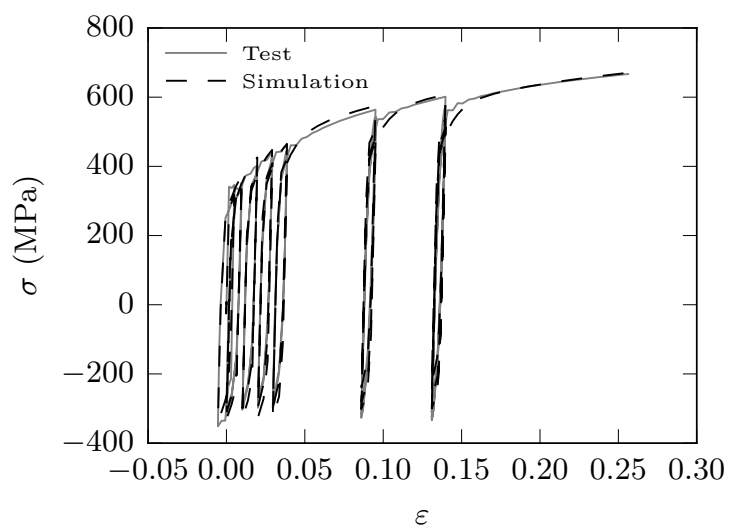

(j) Load protocol \# 10

FIG. 8: (Cont.) Comparison of test results and the combined Voce and Chaboche model with two backstresses estimated with NTR $(\mathrm{J})$ for S355J2+N (t=50mm) steel 

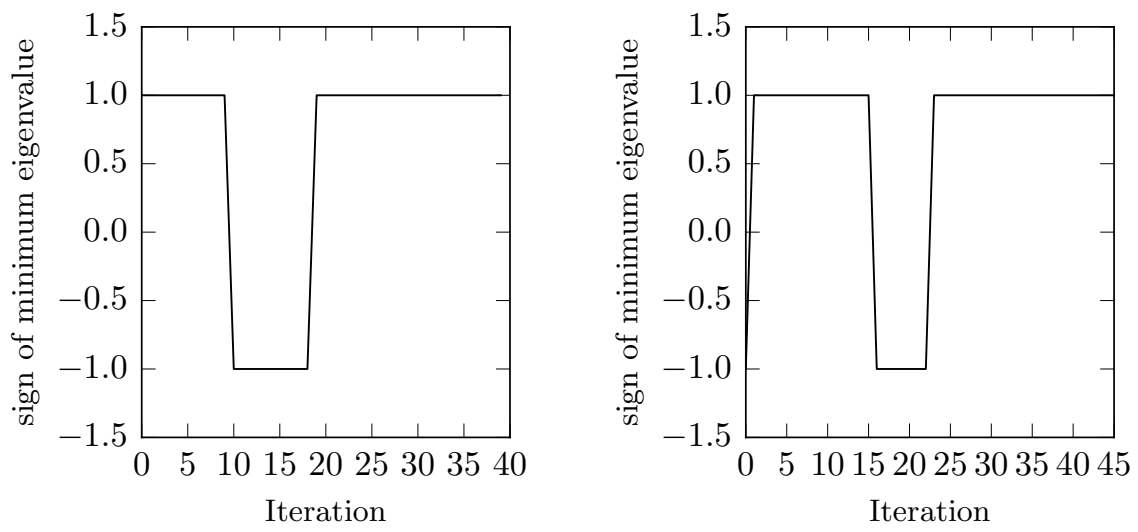

(a) 1 backstress at starting point 1 (b) 1 backstress at starting point 3
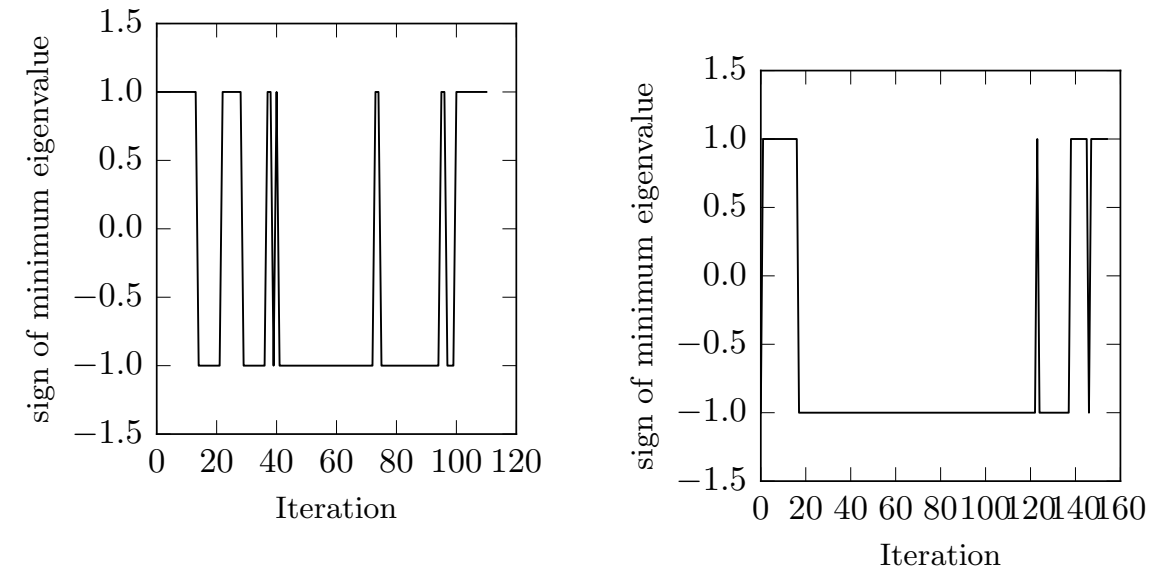

(c) 4 backstresses at starting point 1

(d) 4 backstress at starting point 3

FIG. 9: Evolution of the minimum eigenvalue sign of the Hessians in the NTR with SVD preconditioning method for different starting points and number of backstresses 